\title{
Stabilitätskriterien für Näherungsverfahren bei singulären Integralgleichungen in $L^{p}$
}

\author{
S. Prössdorf und A. Rathsfeld
}

Es werden Näherungsverfahren für eindimensionale singuläre Integralgleichungen mit stückweise stetigen Koeffizienten auf einfachen geschlossenen Ljapunow-Kurven betrachtet. Für Galerkin-Verfahren mit Spline-Funktionen und für Kollokattionsverfahren mit Spline- und trigonometrischen Ansatzfunktionen werden notwendige und hinreichende Kriterien für ihre Stabilität' in $L^{p}$ hergeleitet. Das Kernstück bildet cin allgemeines Stabilitütskriterium für Folgen von Nüherungsoperatoren mit Zirkulantenstruktur.

Рассматриваются приолижениые методы реиения одномерных сингулярных интегральных уравнений с кусочно-непрерывными коэффициентами на простых замкнуых кривых Ляпунова. Для метода Галерюина со сплайн-функцияии и метода коллокаций со сплаін - нли тригонометрическими шункцинми вығодлтся необходимые и достаточныс условыя их устойчивости в $L^{p}$. 【еитральнал часть - обций критериfі устоп̆чивости послелонательности ашироксмирующих операторов, имеющих циркулянтную, структуру.

Approximation methods for one-dimerisional singular integral equations with piecewise continuous coefficients on simple closed Liapunov curves are considered. For spline Galerkin metll. ods and for spline and trigonometric collocation, necessary and sufficient conditions for their

- stability in $L^{p}$ are given. The central idea is a general stability criterion for sequences of approximate operators with circulant structure.

\section{Einleitung}

In der vorliegenden Arbeit werden Näherungsverfahren zur Iösung der Gleichung

$$
\begin{aligned}
& (A x)(t):=a(t) x(t)+b(t) \frac{1}{\pi i} \int \frac{x(\tau)}{\tau-t} d \tau=y(l) \\
& \quad\left(y \in L^{p}(\Gamma), 1<p<\infty\right):
\end{aligned}
$$

untersucht, wobei $\Gamma=\{\gamma(s): s \in[0,1]\}$ eine einfache geschlossene Ijapunov-Kurve; und $a, b$ auf $\Gamma$ vorgegebene stiickweise stetige Funktionen bezeichnen (vgl. Abschnitt. 1.2). Für die gesuchte Funțtion $x \in L^{p}(\Gamma)$ soll eine Näherung $x_{n}$ alls dem Raum der trigonometrischen Polynome $T_{n}^{\prime}=\left\{\sum_{j=-r}^{r} \xi_{j} t^{j}: \xi_{j} \in \mathrm{C}\right\}(n=2 r+1)$ bzw. aus dem SplineRaum $S_{n}{ }^{d}$ bestimmt werden (eine auf $\Gamma$ erklärte Funktion $\varphi$ gehört dem Spline-Raum $S_{n}{ }^{d}$ der Ordnung $d \geqq 0$ an, wenn $\varphi \circ \gamma$ eine $(d-1)$-mal stetig differenzierbare Funktion ist und ihre Einschränkung auf das Intervall $[k / n,(k+1) / n)$ für ungerades $d$ bzw. auf $[(k-1 / 2) / n,(k+1 / 2) / n)$ für gerades $d$ ein Polynom vom Grade kleiner oder gleich $d$ ist). Beim sogenannten $\varepsilon$-Kollokationsverjahren $(-1 / 2<\varepsilon \leqq 1 / 2)$ bestimmt Iman $\dot{x}_{n}$ aus dem Gleichungssystem $\left(A x_{n}\right)(\gamma((k+\varepsilon) / n))=y(\gamma((k+\varepsilon) / n)) .(k=0, \ldots$, $n-1)$, bèin Galerkin-Verfahren mit Spline-Funktionen aus dem Gleichungssystem 
$\left(A x_{n} ; \varphi_{k}^{(n)}\right)=\left(y, \varphi_{k}^{(n)}\right)(k=0, \ldots, n-1)$, wobei $(\cdot ; \cdot)$ das $L^{2}$-Skalarprodukt und $\left\{\varphi_{k}{ }^{(n)}\right\}$ eine Basis des Raumes $S_{n}{ }^{d}$ bezeichnet.

Bekanntlich gehören die genannten Verfahren zur Klasse der Projektionsverfahren, bei denen eine Näherungslösung $x_{n} \in \operatorname{im} K_{n}$ der Operatorgleichung $A x=y$ aus der Gleichung $K_{n} A x_{n}=K_{n} y$ bestimmt wird, wobei $K_{n}$ gegebene Projektoren bezeichnen. Ein Projektionsverfahren heißt stabil, wenn ein $\delta>0$ und ein $n_{0} \in \mathbf{N}$ derart existieren, daß fïr alle $n \geqq n_{0}$ und alle $\varphi^{(n)} \in \operatorname{in} K_{n}$ die Ungleichung $\left\|K_{n} A \varphi^{(n)}\right\|$ $\geqq \delta\left\|\varphi^{(n)}\right\|$ gilt. Aus der Stabilität des Projektionsverfahrens läßt sich, unter gewissen zusätzlichen Voraussétzungen, leicht die Konvergenz der Näherungslösung $x_{n}$ gegen die Lösung $x$ der Gleichung (0.1) ableiten (vgl. Abschnitt 1.1). Deshalb ist es wichtig zu wissen, für welche Operatoren $A$ ein betrachtetes Projektionsverfahren stabil ist. Für die Kollokation mit trigonometrischen Ansatzfunktionen (in diesem Fall kann aus Symmetriegründen $\varepsilon=0$ vorausgesetzt werden) wird im Abschnitt 6.3 gezeigt, da $\beta$ das Verfahren für den Operator $A$ der Gestalt (0.1) im Falle des Einheitskreises $\Gamma_{0}=\left\{\gamma_{0}(s)=\exp (i 2 \pi s): s \in \mathbf{R}\right\}$ genau dann stabil in $L^{p}\left(\Gamma_{0}\right), 1<p<\infty$, ist, wenn $A$ und $A_{-1}:=a l-b S_{\Gamma_{0}}$ (vgl. Abschnitt 1.2) im Raum $L^{p}\left(\Gamma_{0}\right)$ invertierbar sind. Die Notwendigkeit dieser Bedingungen wurde zuerst von Jungranss und SilbBermann [11] (mit anderen Methoden) bewiesen. In [10] wurde für den Spezialfall $p=2$ auch ihre Hinlänglichkeit gezeigt. Für die Stabilität des $\varepsilon$-Kollokationsverfahréns mit Splinc'Funktionen in $L^{p}(T)$ wird im Abschnitt 6.1 eine notwendige und hinreichende Bedingung angegeben. Im Spezialfall $\varepsilon=0$ lautet diese, da $\beta$ für alle $t \in \Gamma$ entweder die Halbgerade $(-\infty, 0]$ den Kreishogen

niit.

$$
\begin{aligned}
K= & \left\{f(\mu) \frac{a(t+0)+b(t+0)}{a(t+0)-b(t+0)}\right. \\
& \left.+(1-f(\mu)) \frac{a(t-0)+b(t-0)}{a(t-0)-b(t-0)}: \mu \in[0,1]\right\}
\end{aligned}
$$

$f(\mu)= \begin{cases}\mu & \text { für } p=2, \\ \sin \left(\pi\left(1-\frac{2}{p}\right) \mu l\right) \exp \left(i \pi\left(1-\frac{2}{p}\right)(\mu-1)\right) / \sin \left(\pi\left(1-\frac{2}{p}\right)\right) \text { für } p \neq 2,\end{cases}$

nicht schneidet oder die Halbgerade $(-\infty, 0)$ obigen Kreisbogen in zwei inneren Punkten schneidet (dabei soll ein Berïhrungspunkt als doppelter Schnittpunkt zählen). Im Fall $p=2$ erhält man hieraus (bzw: aus Satz 6.1 und Satz 6.2) die bekannten Stabilitätsbedingungen von Prössdorf und ȘChMind [15], Arnold und Wexdland [2], Prössdorf und Rathsfeld [13] sowie Schmidt [18]. Im Abschnitt 6.2 wird gezeigt, da $B$ im Falle stetiger Koeffizienten $a, b$ das Galerkin-Verfahren in allen Räumen $L^{p}\left(\Gamma_{0}\right)(1:<<\infty)$ gleichzeitig stabil ist, nämlich genau dann, wenn fïr alle $t \in \Gamma_{0}$ und $\lambda \in[-1,1]$ die Bedingung $\dot{a}(t)+b(t) \lambda+0$ erfüllt ist. Falls $a$ und $b$ stiickweise stetig sind und die Sprungstellen dieser Funktionen zu den Unstetigkeitsstellen der Splines aus $S_{n}{ }^{0}$ gehören, ist das Galerkin-Verfahren für $d=0$ genau dann stabil in $L^{p}\left(I_{0}\right)$, wenn die 0 -Kollokation stabil ist. Im Spezialfall $p=2$ erhält nian die Stabilitätskriterien von Schmidt [20] sowie Prösssorf und RAThSFEld [14].

Beim Beweis der oben genannten Stabilitätskriterien wird gezeigt, daß die Matrizen der Kollokations- bzw. Galerkin-Gleichungssysteme eine einheitliche Struktur besitzen: In Analogie zur Gestalt des Operators $A=a I+b S_{\Gamma_{0}}$ haben sie im wesentlichen die Geštalt $a_{n}+b_{n} Z_{n}$. Dabei sind $a_{n}=\left(a\left(\gamma_{0}((k+\varepsilon) / n)\right) \delta_{k, j}\right)_{k, j}$ und analog $b_{n}$ Diagonalmatrizen und $Z_{n}$ ein Zirkulant der Gestalt

$$
\left(\frac{1}{n} \sum_{s=0}^{n-1} \varrho(\exp (i 2 \pi s / n)) \exp (i 2 \pi s(k-l) / n)\right)_{k . l}
$$


wobei $\varrho$ eine auf $\Gamma_{0}$ erklärte stuickweise stetige Funktion ist, die durch das jeweilige Verfahren festgelegt ist. Die Eigenwerte von $Z_{n}$ sind also Funktionswerte von $\varrho$. Mit Lemma 5.1 wird ein Stabilitätskriterium für Matrixfolgen der Gestalt $\left\{a_{n}+b_{n} Z_{n}\right\}$ bewiesen, aus dem sich leicht die einzelnen Stabilitätsbedingungen für die Kollokations- und Galerkin-Verfahren ableiten lassen. Letzteres stellt eine Verallgemeinerung von [13: Theorem 0.1] dar.

\section{Abstrakte Näherungsverfahren und singuläre Integraloperatoren}

1.1 Es sei $X$ ein Banach-Raum, $\mathscr{L}(X)$ die Menge aller stetigen linearen Operatoren in $X$ und $A \in \mathscr{L}(X)$ invertierbar. Die Gleichung $A x=y(y \in X)$ soll näherungsweise gelöst werden. Dazu sei in $X$ eine Folge beschränkter Projektoren $L_{n}$ gegeben, die stark gegen die identische Abbildung konvergiert. Außerdem soll eine Folge von Näherungsoperatoren $A_{n} \in \mathscr{L}\left(\mathrm{im} L_{n}\right)$ derart gegeben sein, daß $A_{n} L_{n}$ stark gegen $A$ konvergiert. Aus der Gleichung $A_{n} x_{n}=y_{n}\left(y_{n} \in\right.$ im $\left.L_{n}\right)$ bestimnit man dann eine Näherungslösung $x_{n} \in$ in $L_{n}$. Ein solches Näherungsverfahren .heißt konvergent, wenn ein $n_{0} \in \mathbf{N}$ derart existiert, daß die Gleichung $A_{n} x_{n}=y_{n}$ für alle $n \geqq n_{0}$ und alle $y_{n} \in$ im $L_{n}$ eindeutig lösbar ist und wenn für alle $y \in X$ und alle Folgen $\left\{y_{n}\right\} \subset \operatorname{im} L_{n}$ mit $y_{n} \rightarrow y$ die entsprechende Folge der Näherungslösungen $x_{n}$ gegen $x=A^{-1} y$ konvergiert. Das Näherungsverfahren mit den Operatoren $A_{n}$ bzw. die Operatorfolge $\left\{A_{n}\right\}$ heißt stabil, wenn ein $n_{0} \in \mathbf{N}$ derart existiert, daß $A_{n}$ für $n \geqq n_{0}$ invertierbar ist und sup $\left\|A_{n}{ }^{-1}\right\|<\infty$ gilt. Bekanntlich konvergiert das Näherungsverfahren genau dann; wenn es stabil ist (vgl. [8]). Projektionsverfahren können als Spezialfall solcher allgemeinen Näherungsverfahren betrachtet werden. In diesem Fall wählt man noch eine Folge von Projektoren $K_{n}$ im Raum $X$ mit im $K_{n}=\operatorname{im} L_{n}$ und setz.t. $A_{n}^{\prime}=\left.K_{n} A\right|_{\mathrm{im} L_{n}}$ sowie $y_{n}=K_{n} y$.

1.2 Im folgenden sollen Näherungsverfahren zur Lösung einer singulären Integralgleichung der'Gestalt (0.1) auf einer Kurve $\Gamma$ untersucht werden. Dábei sei $\Gamma$ eine einfache geschlossene Ljapunow-Kurve in der komplexen Ebene C. Die Kurve besitzt also eine 1-periodische Parameterdarstellung $\gamma: \mathbf{R} \rightarrow \mathbf{C}$ mit einer stetigen, nirgends verschwindenden Ableitung $\gamma^{\prime}$. Es soll im weiteren yorāusgesetzt werden, da $\beta$ $\gamma^{\prime}$ einer Lipschitz-Bedingung genügt und $\Gamma$ positiv orientiert ist.

Auf $\Gamma$ werden nun verschiedene Funktionenräume betrachtet. Der Grundraum $\ddot{X}$ unserer singulären 'Integralgleichung sei $L^{p}(\Gamma)(1<p<\infty)$. Wenn

$$
\hat{f}(k)=\int_{-1 / 2}^{1 / 2}(f \dot{\circ} \gamma)(x) \exp (-i 2 \pi k x) d x
$$

den $k$-ten Fourier-Koeffizienten einer auf $\Gamma$ erklärten Funktion $f$ bezeichnet, dann sei $F^{\prime} l^{p}$ der Banach-Raum aller auf $\Gamma_{0}$ definierten 'Funktionen $f$, deren Norm $\|f\|_{F l^{p}}$ $=\left(\sum_{k \in Z}|\hat{f}(k)|^{p}\right)^{1 / p}$ endlich ist. Die Räume aller stetigen und aller stiickweise stetigen Funktionen auf $\Gamma$ sollen mit $C(\Gamma)$ bzw. $P C(\Gamma)$ bezeichnet werden. Dabei heißt $f$. stïckweise stetig, wenn $f$ bis auf endlich viele Punkte stetig ist und in allen Punkten $t \in \Gamma$ endliche einseitige Grenzwẹrte $f(t+0)=: f(t)$ und $f(t-0)$ existieren. Die abgeschlossene Hülle von $P C(\Gamma)$, (bezïglich der Supremumnorm) soll mit $\overline{P C}(\Gamma)$ bezeichnet werden. Ferner sei $P Q^{2}(\dot{\Gamma})$ die Menge aller $f \in P C(\Gamma)$, die außerhalb der Sprungstèllen zweimal stetig differenzierbar sind und derén zweite Ableitung zu $P C(\Gamma)$ gehört.

In den Räumen $L^{p}(\dot{\Gamma})$ bzw' $F l^{p}(1<p<\infty)$ kann mạn den singulären Integraloperator $A=a I+b S_{r}$ betrachten, wobei $a$ und $b$ die Operatoren' der Multiplikation 
mit den Funktionen $a, b \in \overline{P C}(\Gamma)$ bzw. $a, b \in P C^{2}\left(\Gamma_{0}\right)$ bezeichnen und $S_{\Gamma}$ der durch

$$
\left(S_{\Gamma} x\right)(t)=\frac{1}{\pi i} \int_{i} \frac{x(\tau)}{\tau-t} d \tau \quad(t \in \Gamma)
$$

definierte Cauchysche singuläre Operator ist. Mit den Bezeichnungen $c=a+b$, $d=a-b, P_{r}=\left(I+S_{r}\right) / 2$ und $Q_{r}=I-P_{r}$ läßt sich $A$ in $\operatorname{der}$ Form $A=c P_{r}$ $+d Q r$ schreiben. Bekanntlich ist $A \in \mathscr{L}\left(L^{p}(\Gamma)\right)$ genau dann ein Noetherscher Operator (auch Fredholmscher Operator genannt), wenn für alle $t \in \Gamma$ der Kreisbogen $K$ aus (0.2) nicht durch 0 verläuft. Der Operator $A \in \mathscr{L}\left(L^{p}(\dot{\Gamma})\right)$ ist invertierbar, wenn zusätzlich der funktionentheoretische Index der auf natürliche Weise orientierten Kurve, die durch Vereinigung der Punktmenge $\left\{\left(d^{-1} c\right)(t): t \in I\right\}$ und der die Sprünge der Funktion $d^{-1} \bar{c}$ verbindenden Kreisbögen $K$ entsteht, gleich 0 ist (vg̀l. [9]). Der Operator $A=a I+b S_{\Gamma_{0}} \in \mathscr{L}\left(F l^{p}\right)$ ist genau dann invertierbar, wenn der Operator $A \in \mathscr{L}\left(L^{q}\left(I_{0}\right)\right)(1 / p+1 / q=1)$ invertierbar ist (vgl. [9: Kap. XIV]).

\section{Zirkulanten}

Im Zusammenhang mit den in der Einleitung beschriebenen Näherungsverfahren sowie mit den Projektoren, die im Abschnitt 3 betrachtet werden sollen, treten verschiederie Zirkulanten auf. Eine Matrix $\left(a_{j, k}\right)_{j, k}^{n-1}$ heißt Zirkulant, wenn die Elemente jeder zur Hauptdiagonalen parallelen Diagonalen einander gleich sind und wenn ferner die Elemente der $k$-ten Diagonale über der Hauptdiagonale $(k=1, \ldots, n-1)$ mit den Elementen der $(n-k)$-ten Diagonale unter der Hauptdiagonale übereinstimmen. Ziel dieses Abschnittes ist es, für spezielle Zirku. lantenfolgen eine geeignete Normabschätzung (Lemma 2.1) hèrzuleiten.

2.1 Im weiteren sei`der Einfachheit halber $n \neq 2 r+1$ vorausgesetzt. (Der Fall $n=2 r$ kann völlig analog behandelt werden.) Es soll mit $l^{n}(n)$ der komplexe $n$-dimensionale $l^{p}$-Raum, mit $\|\cdot\|_{p}$ dessen Norm und mit $\xi=\left(\xi_{-r}, \ldots, \xi_{r}\right)^{\mathrm{T}}$ dessen Elemente bezeichnet werden. Für einen Operator $A_{n} \in \mathscr{L}\left(l^{p}(n)\right)$, der offenbar mit einer Matrix aus $\mathbf{C}^{n \times n}$ identifiziert werden kann, soll $\left\|A_{n}\right\|_{p}$ die ibliche Operatornorm bezeichnen. Ana$\log$ zu [12: Kap. VI] führt man in $l^{p}(n)$ dic diskrete Fourier-Transformation $U$ und ihre Inverse $U^{-1}$ ein:

$$
(U \xi)_{k}=\frac{1}{\sqrt{n}} \sum_{l--r}^{r} \exp \left(\frac{i 2 \pi k l}{n}\right) \xi_{l}, \text { und },\left(U^{-1} \xi\right)_{k}=\frac{1}{\sqrt{n}} \sum_{l=-r}^{r} \exp \left(\frac{-i 2 \pi k l}{n}\right) \xi_{l} .
$$

Für $\xi \in \mathbf{C}^{n}$ 'soll $M_{\xi}$ den Multiplikationsoperator $\left(\delta_{;, k} \xi_{k}\right) \in \mathscr{L}\left(l^{p}(n)\right)$ und $C_{\xi}$ den Faltungsoperator $\left(\xi_{k-l}\right) \in \mathscr{L}\left(l^{p}(n)\right)$ bezeichnen, wobei $k-l$ diejenige Zahl aus $\{-r, \ldots, r\}$ ist, die sich von $k-l$ nur um ein ganzzahliges Vielfaches von $n$ unterscheidet. Die Matrix $M_{\xi}$ ist also eine Diagonalmatrix und $C_{\xi}$ ein Zirkulant. Für $A_{n} \in \mathscr{L}\left(l^{p}(n)\right)$ sei $\hat{A}_{n}=U A_{n} U^{-1}$. Wie man leicht sieht, ist $A_{n}$ genail dann ein Zirkulant, wenn es cin $\xi \in \mathbf{C}^{n}$ mit $A_{n}=U M_{\xi} U^{-1}$ gibt. Es gilt $C_{\eta}=\hat{M}_{\xi}$ genau dann, wenn $\hat{\eta}=n^{-1 / 2} U \xi$, d. h. $\xi=n^{+1 / 2} U^{-1} \eta$ ist. In Analogie zu den Faltungsoperatoren auf der reellen Achse erhält man die Youngsche Ungleichung $\left\|C_{\xi}\right\|_{p} \leqq\|\xi\|_{11}\left(\xi \in l^{1}(n)\right)$.

2.2 In den folgenden Abschnitten treten Zirkulantén $\hat{\varrho}_{n}$ auf, deren Eigenwerte Funktionswerte einer Funktion $\varrho \in \overline{P C}\left(\Gamma_{0}\right)$ sind :

$$
\varrho_{n}:=\left(\varrho(\exp (i 2 \pi k / n)) \delta_{j, k}\right)_{j, k--r}^{r}, \quad \hat{\varrho}_{n}=U \varrho_{n} U^{-1}
$$


Lemma 2.1: Es sei $1<p<\infty$. Dann gibt es eine Konstante $C>0$ derari, da $\beta$ für alle Funktionen $\varrho \in \overline{P C}\left(\Gamma_{0}\right)$ mit beschränkter Variation Var $\varrho$ die Abschätzung $\left\|\hat{Q}_{n}\right\|_{p}$ $\leqq C\{\sup \mid \varrho(t)\}+\operatorname{Var} \varrho\}$ erfüllt ist.

Beweiș: a) Zuerst sei $\varrho$ die charakteristische Funktion des Kreisbogens zwischen zwei beliebigen festén Punkten auf $\Gamma_{\mathbf{0}}$. Offensichtlich kann vorausgesetzt werden, daß - 1 kein innerer Punkt des Trägers supp $\varrho$ ist. Wenn $P_{n} \in \mathscr{L}\left(L^{p}\left(\Gamma_{0}\right)\right)$ durch $P_{n}\left(\sum_{k \in Z} \hat{f}(k) t^{k}\right)=\sum_{k=-r}^{r} \hat{f}(k) t^{k}$ definiert wird, dann gibt es zwei von $n$ abhängige Zahlen $u, v \in \mathbf{Z}$ derart, daB der Operator $\left.P_{n} t^{-u} Q r_{0} t^{u} t^{v} P_{\Gamma_{0}} t^{-v}\right|_{\operatorname{im} P_{n}} \overline{\mathcal{L}}\left(\mathrm{im} P_{n}\right)$ in der Basis $\left\{t_{i=-r}^{i}\right.$ die Matrix $\varrho_{n}$ hat. Der gleiche Operator besitzt.beziuglich der Basis

$$
\left\{\psi_{k}\right\}_{k=-r}^{r}, \quad \psi_{k}(t)=\frac{1}{n} \sum_{j=-r}^{r} \exp (-i 2 \pi k j / i n) l^{\prime},
$$

die Matrix $\hat{\varrho}_{n}$. Aus $\psi_{k}(\exp (i 2 \pi j / n))=\delta_{k, j}(k, j=-r, \ldots, r)$ und [23: Kap. X/(7.6), (7.7)] folgt die Existenz einer Konstanten $D>0$, so da $B$

$$
D^{-1} n^{-1 / p}\|\xi\|_{p} \leqq\left\|\sum_{k=-r}^{r} \xi_{k} \psi_{k}\right\|_{L^{D}\left(\Gamma_{0}\right)} \leqq D n^{-1 / p}\|\xi\|_{p} \quad\left(\xi \in l^{p}(n)\right)
$$

gilt. Folglich ist die mit, $D^{2}$ multiplizierte $O$ peratornorm eines Operators aus $\mathscr{L}\left(\mathrm{im} P_{n}\right.$ ) größer oder gleich der $\mathscr{L}\left(l_{i}^{p}(n)\right)$-Norm seiner Matrix bezüglich der Basis $\left\{\psi_{k}\right\}$. Weil aber die Operatoren $\left.P_{n} t^{-u} Q_{r_{0}}{ }^{u} t^{v} P_{r_{0}} t^{-v}\right|_{i m P_{n}}$ für alle $u, v \in \mathbf{Z}$ und $n \in \mathbf{N}$ gleichmäßig beschränkt sind, gibt es eine von der charakteristischen Funktion 巳 unabhängige Konstante $C>0$ mit $\left\|\hat{o}_{n}\right\|_{p}<C$.

b) Es sei jetzt $\varrho$ eine stiickweise konstante Funktion, d. h., fiir alle $t$ aus dem Kreisbogen zwischen $\exp \left(i 2 \pi s_{k}\right)$ und $\exp \left(i 2 \pi s_{k+1}\right)\left(0=s_{0}<s_{1} \cdots<s_{y}=1\right)$ sei $\varrho^{\prime}(t)$ $=\beta_{k} \in \mathbf{C}$. Wenn $\psi^{k}(k=1, \ldots, N)$ die charakteristische Funktion des. Bogens zwischen 1 und $\exp \left(i 2 \pi s_{k}\right)$ bezeichnet, dann gilt

$$
\begin{aligned}
& \varrho=\sum_{k=1}^{N-1}\left(\beta_{k-1}-\beta_{k}\right) \psi^{k}+\beta_{N^{\prime}-1} \psi^{N}, \\
& \left\|\hat{\varrho}_{n}\right\|_{p} \leqq\left\{\sum_{k=1}^{N-1}\left|\beta_{k}-\beta_{k-1}\right|+\left|\hat{\beta}_{N-1}\right|\right\}_{k=1 \ldots \ldots .}\left\|\left(\psi^{k}\right)_{n} \wedge\right\|_{p} .
\end{aligned}
$$

Aus a) folgt $\left\|\underline{\hat{o}}_{n}\right\|_{p} \leqq C\{\sup |\underline{Q}(l)|+\operatorname{Var} \varrho\}$.

c) Schließlich sei $\varrho \in \overline{P C}\left(\Gamma_{0}\right)$ eine beliebige Funktion mit beschränkter Variation. Dann existiert eine Folge $\left\{Q^{k}\right\}$ stückweise konstanter Funktionen, die für $k \rightarrow \infty$ in der Supremümnorm gegen $\varrho$ konvergiert und fiir die $\operatorname{Var} \varrho^{k}<\operatorname{Var} \varrho$ gilt: Für jedes feste $n$ ind $k \rightarrow \infty$ konvergieren die Operatoren $\left(g^{k}\right)_{n}^{\wedge}$ gegen $\hat{\varrho}_{n}$. Mithin ergibt sich,

$$
\left\|\underline{\hat{o}}_{n}\right\|_{p} \leqq \lim _{k \rightarrow \infty} \sup \left\|\left(\varrho^{k}\right)_{n}^{\wedge}\right\|_{p} \leqq C\{\sup |\varrho(l)|+\operatorname{Var} \varrho\}
$$

\section{Spline-Räume und Projektoren}

In diésem Abschnitt werden Interpolations- und Orthoprojektoren auf die Spline-Räume $S_{n}{ }^{d}$ unter'sucht. Für diese Projektoren lassen sich unter Ver'wendung der im Abschnitt 2.2 betrachteten Zirkulanten gewisse explizite Darstellungsformeln angeben. Mit diesen wird dann die starke Konvergenz der Projektoren gezeigt und im nächsten Abschnitt die DiagonalZirkulanten-Struktur.der Matrizen beim Kollokatious- und Galerkin-Verfahren nachgewiesen. 
3.1 Im Raum $S_{n}{ }^{d}$ ]äßt sich eine Basis $\left\{\varphi_{k}{ }^{(n)}\right\}$ wie folgt angeben (vgl. [3: Section 4.2]): Frklärt man die Faltung $f * g$ zweier auf $\mathbf{R}$ definierter Funktionen $f$ und $g$ als Integral

$$
(f * g)(t)=\frac{1}{\sqrt{2 \pi}} \int_{\mathbf{R}}^{-} f(t-s) g(s) d s \quad(t \in \mathbf{R}),
$$

ist $\Pi^{0}$ die aùf $\mathbf{R}$ erklärte charakteristische Funktion des Intervalls $[-1 / 2,1 / 2]$ und $\Pi^{d}$ die $(d+1)$-fache. Faltung yon $\Pi^{0}$, so sei

$$
\dot{\varphi}_{k}{ }^{(r)}(\gamma(s))=\Pi^{d}(s n-k) \quad(k \in\{-r, \ldots, r\}, \quad s \in[-1 / 2+k / n, 1 / 2+k / n]) .^{\prime},
$$

Lem ma 3.1 [5]: Es sei $1<p<\infty$. Dann gibt es eine Konslante $C \geq 0$ derart, da $\beta$. für alle $n \in \mathbf{N}$ und alle $\xi \in l^{p}(n)$ gilt

$$
C^{-1} n^{-1 / p}\|\xi\|_{p} \leqq\left\|\sum_{j=-\tau}^{r} \xi_{j} \varphi_{j}^{(n)}\right\|_{L^{(i)}} \leqq C n^{-i / p}\|\xi\|_{p} .
$$

3.2 Fïr zwei Funktionen $\dot{f}, g \in L^{2}(\Gamma)$ soll das Skalarprodukt'durch

$$
(f, g)=\int_{-1 / 2}^{1 / 2} f(\gamma(s)) \overline{g(\gamma(s))} d s
$$

definiert sein. Den Orthoprojektor $L_{n}$ des Raumes $L^{2}(\Gamma)$ auf $S_{n}{ }^{d}$ kann man auch im Raum $L^{p}(\Gamma)(1<p<\infty)$ definieren: Für $f \in L^{p}(\Gamma)$ soll $L_{n} f \in S_{n}^{\text {id }}$ diejenige Funktion sein, die für alle $\varphi^{(n)} \in S_{n}^{d}$ die Gleichung $\left(L_{n} f, \varphi^{(n)}\right)=\left(f, \varphi^{(n)}\right)$ erfüllt, Um eine explizite Formel für $L_{n}$ zu erhalten, setzt man $L_{n} f=\sum_{k=-r}^{r} \xi_{k} \dot{\varphi}_{k}^{(n)}$ und $\eta_{k}=\left(f, \varphi_{k}{ }^{(n)}\right)$. Dann
folgt aus der Definition von $L_{n}$

$$
\left(\left(\varphi_{k}^{(n)}, \varphi_{j}^{(n)}\right)\right)_{j, k--r}^{r} \xi=\eta, \quad \xi \stackrel{\prime}{=}\left(\xi_{-r}, \ldots, \xi_{r}\right)^{\mathrm{T}}, \quad \eta \doteq\left(\eta_{-r}, \ldots, \eta_{\mathrm{r}}\right)^{\mathrm{T}} .
$$

Die Elemente $\left(\varphi_{k}^{(n)}, \varphi_{j}^{(n)}\right)$ der Gramschen Matrix lassen sich iiber die Fourier-Koeffizienten bestimmen. Fs gilt

$$
\left(\varphi_{k}^{(n)}, \varphi_{j}^{(n)}\right)=\sum_{l \in \mathbf{Z}}\left(\varphi_{k}^{(n)}\right)^{\wedge}(l) \overline{\left(\varphi_{j}^{(n)}\right)^{\wedge}(l)} .
$$

Unter Benutzung der fiir alle $\bar{p}, k \in \mathbf{Z}$ und $\varphi^{(n)} \in S_{n}{ }^{d}$ in [1] bewiesenen Formel

$$
\left(\varphi^{(n)}\right)^{\wedge}(p+k n)=(-1)^{k(d+1)}\left(\varphi^{(n)}\right)^{\wedge}(p) p^{d+1} /(p+k n)^{d+1}
$$

und der fïr $u \in\{=r, \ldots, r\}$ gültigen Bezichung

$$
\begin{aligned}
& \left(\varphi_{u}{ }^{(n)} \circ \gamma\right)(s)=\left(\varphi_{0}{ }^{(n)} \circ \gamma\right)(s-u / n), \\
& \left(\varphi_{u}{ }^{(n)}\right)^{\wedge}(k)=\exp \frac{-i 2 \pi k u}{n}\left(\varphi_{0}{ }^{(n)}\right)^{\wedge}(k)
\end{aligned}
$$

erhält man .

$$
\left(\varphi_{k}^{(n)}, \varphi_{j}^{(n)}\right)=\frac{1}{n^{2}} \sum_{s=0}^{n-1} \exp (i 2 \pi s(j-k) / n) \alpha_{i}(\exp (i 2 \pi s / n))
$$


mit:

$$
\begin{aligned}
& \alpha(\exp (i 2 \pi s))_{i}:=\left|\left(\Pi^{d}\right)^{\wedge}(s)\right|^{2} s^{2(d+1)} \sum_{k \in \mathbb{Z}^{-}}(s+k)^{-2(d+1)}, \\
& \left(\Pi^{d}\right)^{\wedge}(s):=\int_{\mathbf{R}} \exp (-i 2 \pi y s) \Pi^{d}(y) d y
\end{aligned}
$$

Die Gramsche Matrix $\left(\left(\varphi_{k}^{(n)}, \varphi_{j}^{(n)}\right)\right)$ ist also ein Zirkulant und hat die Gestalt $n^{-1} \hat{\alpha}_{n}$. Wenn $[\cdot, \cdot]$ das übliche Skalarprodukt des Raumes $l^{2}(n)$ bezeichnet, dann folgt aus Iemma 3.1

$$
[\zeta, \zeta] \leqq C^{2} n\left(\sum_{k=-r}^{r} \zeta_{k} \varphi_{k}^{(n)}, \quad \sum_{j=-r}^{r} \zeta_{j} \varphi_{j}^{(n)}\right)=C^{2}\left[\hat{\alpha}_{n} \zeta, \zeta\right] \cdot \quad\left(\zeta \in \dot{l}^{2}(n)\right) .
$$

Somit genügen die Eigenwerte von $\hat{\alpha}_{n}$, d. h. die Funktionswerte $\alpha(\exp (i 2 \pi k / n))$ $(k=0, \ldots, n-1)$, der Abschätzung $C^{-2} \leqq|\alpha(\exp (i 2 \pi k / n))|$. Die Funktion $\alpha$ besitzt also auf $\Gamma_{0}$ keine Nullstellen, und aus (3.1) erhält man als gesuchte Darstellung fiir den Projektor $L_{n}$

$$
\xi=n\left(\alpha^{-1}\right)_{n}^{\wedge} \eta \text {. }
$$

Lemma 3.2 (vgl. [4]): Die Folge $\left\{L_{n}\right\}$ ist in $L^{p}(\Gamma)(1<p<\infty)$ gleichmäßig beschräńkt und konvergiert stark gegen den identischen Operator.

Beweis: Aus der Hölderschen Ungleichung und IL I emma 3.1 folgt für eine geeignete Konstante $C>0$.

$$
\begin{aligned}
& \left|\eta_{k}\right| \leqq\left\|\left.(f \circ \gamma)\right|_{\left(\frac{k-(d+1) / 2}{n}, \frac{k+(d+1) / 2}{n}\right)}\right\|_{L^{p}}\left\|\left(\varphi_{k}^{(n)} \circ \gamma\right)\right\|_{L^{\sigma}} \leq C n^{-1 / q}\left\|\left.(f \circ \gamma)\right|_{(\ldots \ldots .)}\right\|_{L^{\nu}}, \\
& \|\eta\|_{p} \leqq C n^{-1 / q}\|f\|_{L^{p}(\Gamma)}
\end{aligned}
$$

Hieraus ergibt sich zusammen mit (3.5), Lemma 2.1 und Lemma 3.1

$$
\begin{aligned}
\left\|L_{n} f\right\|_{L^{D}(\Gamma)} & =\left\|\sum_{k=-r}^{r} \xi_{k} \varphi_{k}\right\|_{L^{D}(\Gamma)} \leqq C n^{-1 / p}\|\xi\|_{p} \\
& \leqq C n^{1-1 / p} \cdot\left\{\operatorname{Var} \alpha^{-1}+\sup _{t \in \Gamma_{0}}\left|\alpha^{-1}(t)\right|\right\}\|\eta\|_{p} \leqq C\|f\|_{L^{p}(\Gamma)} .
\end{aligned}
$$

Die Folge $\left\{L_{n}\right\}$ ist also in allen Räumen $L^{p}(\Gamma)(1<p<\infty)$ gleichmäßig beschränkt. In $L^{2}(\Gamma)$ folgt die starke Konvergenz aus [7: Theorem 1]. Unter Benutzung der Höiderschen Ungleichung ergibt sich aus ihr die $L^{p}$-Konvergenz

3.3 Der Interpolationsprojektor $K_{n}$ mit dem Bildraum $S_{n}^{d}$ wird für Riemann-integrierbare Funktionen $f$ durch $\left(K_{n} f\right)(\gamma((k+\varepsilon) / n))=f(\gamma((k+\varepsilon) / n))(k=-r, \ldots, r)$. erklärt. Für alle $\varepsilon \in(-1 / 2,1 / 2)$ ist in [19] die Existenz dieses Projektors gezeigt. Um wieder eine explizite Formel herzuleiten, setzt man $K_{n} f=\sum_{k=-r}^{r} \xi_{k} \varphi_{k}^{(n)}$ und $\eta_{k}$
$=f\left(\gamma\left(\left(k_{-}+\varepsilon\right) / n\right)\right)$. Dannfolgt

$$
\left(\varphi_{k}^{(n)}(\gamma((j+\varepsilon) / n))\right)_{j, k=-r}^{r} \xi=\eta \text { ' }
$$

Die Elemente der hier links stehenden Matrix können nun ïber die Fourier-Reihe berechnet werden. Es ergibt sich

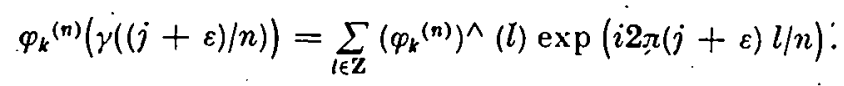


Unter Ausnutzung von (3.2) und (3.3) erhälț man

$$
\varphi_{k}^{(n)}(\gamma((j+\varepsilon) / n))=\frac{1}{n} \sum_{s=0}^{n-1} \exp (i 2 \pi s(j-k) / n) \varrho(\exp (i 2 \pi s / n))
$$

mit, für $s \in(0,1)$,

$$
\varrho(\exp (i 2 \pi s))=\left(\Pi^{d}\right)^{\wedge}(s) s^{d+1} \exp (i 2 \pi \varepsilon s) \sum_{k \in Z} \frac{\exp (i \pi k(2 \varepsilon+d+1))}{(s+k)^{d+1}}
$$

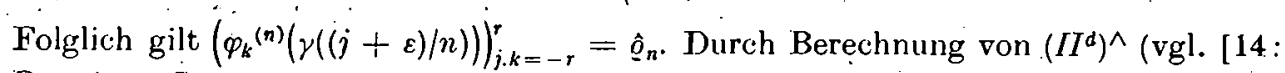
Beweis zu Iemma 2.1]) sieht man, daß $\varrho$ auf $\Gamma_{0}$ keine Nullstellen besitzt (vgl. [19]). Aus (3.6) ergibt sich nun

$$
\xi=\left(\varrho^{-1}\right)_{n} \wedge \eta
$$

Lemma 3.3: - Für alle Riemann-integrierbaren Funktionen ' $f$ auf $\Gamma$ gilt $K_{n} f \rightarrow f$ $(n \rightarrow \infty)$ in der Norm von $L^{p}(I)(1<p<\infty)$.

Beweis: Analog zu [15: Lemma 2.1] zeigt man $f-D_{r}^{-1} \sum_{k=\tau r}^{r} \eta_{k} \varphi_{k}^{(n)} \rightarrow 0$, wobei $D$. die von $\varepsilon, j$ und $n$ unabhängige Summe $\sum_{k=-r}^{r} \varphi_{k}{ }^{(n)}(\gamma((j+\varepsilon) / n))$ bezeichnet. Es mu $B$ also
nur noch

$$
K_{n} f-D^{-1} \sum_{k=-r}^{r} \eta_{k} \varphi_{k}^{(n)} \rightarrow 0, \quad \text { d. h. } \quad n^{-1 / p}\left\|\left(\varrho^{-1}\right)_{n}^{\wedge} \eta-D^{-1} \eta\right\|_{p} \rightarrow 0
$$

'bewiesen werden. Nach Lemma 2.1. genügt es,

$$
n^{-1 / p}\left\|\eta-D^{-1} \hat{\varrho}_{n} \eta\right\|_{p} \rightarrow 0, \quad \text { d. h. } \quad \sum_{k=-r}^{r}\left(\eta_{k}-D^{-1}\left(\hat{o}_{n} \eta\right)_{k}\right) \varphi_{k}^{(n)} \rightarrow 0
$$

zu zeigen. Letzteres beweist man analog zu [15: Lemma 2.1]. Dabei ist zu beachten, daß in jeder Zeile, von $\hat{\varrho}_{n}$ nur $d$ von 0 verschiedene Elemente stehen

\section{Die Näherungsoperatoren für Spline-Methoden}

Das Ziel dieses Abschnittes ist es, die Matrizen der Näherungsoperat toren für singuläre Integraloperatoren zu bestimmen, die bei Kollokations- und Galerkin-Verfahren mit Spline-Ansatzfunktionen auftreten. Außerdem soll hier die starke Konvergenz dieser Näherungsoperatoren gegen den singulären Integraloperator in $L^{p}(\Gamma)(1<p<\infty)$ bewiesen werden. Wenn $K_{n}$ und $L_{n}$ die in $A$ bschnitt 3 definierten Projektoren bezeichnen, dann läßt sich das Niherungssystem des Kollokations-bzw. des Galcrkin-Verfahrens als Operatorgleichung der Form $K_{n} A x_{n}=K_{n} y$ bzw. $L_{n} A x_{n}=L_{n} y$ schreiben. Fs müssen also die Näherungsoperatoren $\left.K_{n} A\right|_{s_{n}{ }^{d}} \in \mathscr{L}\left(S_{n}{ }^{d i}\right)$ und $L_{n} A \mid \dot{S}_{n} \dot{d} \in \mathscr{L}\left(S_{n} \dot{c}\right)$ untersucht werden.

4.1. Zuerst sollen die Näherungsoperatoren für den Multiplikationsoperator untersucht werden. Es sei $a \in \overline{P C}(\Gamma)$. Dann folgt aus (3.7); daß $\left.K_{n} a\right|_{s_{n} \text { a }}$ in der Basis $\left\{\varphi_{k}^{(n)}\right\}$ die Matrix

$$
\left(Q^{-1}\right)_{n} \wedge a_{n} \hat{\varrho}_{n} \quad \text { mit } \quad a_{n}=\left(\delta_{k, l} a(\gamma((k+\varepsilon) / n))\right)_{k, l=-r}^{r}
$$

besitzt. (Für lateinische Buchstaben, z. B. $a$, soll die Matrix $a_{n}$ wie in (4.1) erkلärt sein. Für griechische Buchstáben, z. B. $\varrho$, soll $\varrho_{n}$ wie in Abschnitt 2.2 definiert verden.) 
Lemma 4.1: Es sei $a \in \overline{P C}(\Gamma)$. Die Operatoren $\left.K_{n}{ }_{n}^{\prime}\right|_{S_{n}{ }^{d}} \operatorname{sind}$ in $L^{p}(\Gamma)(1<p<\infty)$ gleichmäßig beschränkt, und $\left\{K_{n} a L_{n}\right\}$ konvergiert stark geg̀en $a$.

Bew'eis: Aus (4.1), $\left\|a_{n}\right\|_{p} \leqq \sup \{|a(\tau)|: \tau \in \Gamma\}$, Lemma 2.1 und Lemma 3.1 folgt die gleichmäßige Beschränktheit. Es genügt also, die Konvergenz $K_{n} a L_{n} f \rightarrow a f$ für stetige Funktionen $f$ zu beweisen.

Fiir $k, l \in \mathbf{Z}$ soll $k-l \in\{-r, \ldots, r\}$ wieder diejenige $Z$ ahl sein, die sich von $k-l$ nür um ein ganzzahliges Vielfaches von $n$ unterscheidet. Es sei $\varrho \in F l^{1}$. Dänn hängt das Element der $k$-ten Zeile und $l$-ten Spalte des Zirkulanten $\hat{\varrho}_{n}$ nur von $k-l$ ab und soll in weiteren mit $\hat{\varrho}_{n, k} \sim 1$ bezeichnet werden. Falls $\varrho$ eine Potenzfunktion $\varrho(t)=t^{u}(u \in \mathbf{Z})$ ist, dann gilt $\hat{\varrho}_{n, k}=1$, wenn $u+k$ ein Vielfaches von $n$ ist, und $\hat{\varrho}_{n, k}=0$, wenn $u_{1}+k$ nicht durch $n$ teilbar ist. Es ergibt sich

$$
\hat{e}_{n, k}=\sum_{q \in \mathbf{Z}} \hat{\varrho}(n q-k), . \quad \text { falls } \quad \varrho(l)=\sum_{s \in Z} \hat{o}(s) l^{s} \in F l^{1}
$$

Aus dieser Formel und der Youngschen Ungleichung erhält man $\left\|\hat{o}_{n}\right\|_{\infty} \leqq \| \hat{\varrho}_{F /}$. Zusammen mit dieser Abschätzung folgt aus dem Beweis zu Lemma 3.2, daß die Folge, $\left\{L_{n}\right\}$ auch in den mit der Supremumnorm versehenen Raım $C(\Gamma)$ gleichmäBig beschränkt ist. Für $d \geqq 1$ ergibt sich aus [7: Theorem 1] die starke Konvergenz von $\left\{L_{n}\right\}$ gegen den identischen Operator des Raumes $C(\Gamma)$. Hieraus schließt man zu-' sammen mit der Beschränktheit des Multiplikationsoperators $a \in: \mathscr{L}(\overline{P C}(\Gamma))$ und der starken Konvergenz von $\left\{K_{n}\right\}$ gegen den Einbettungsoperator von $\overline{P C^{\prime}}(\Gamma)$ in $L^{p}(\Gamma)$ (vgl. Lemma 3.3); daß $\left\{K_{n} a L_{n}\right\}$ stark gegen den Operator $a: C(\Gamma) \stackrel{\rightarrow}{\rightarrow} L^{p}(\Gamma)$ konvergiert. Für $d=0$ folgt die Behauptung des Lemmas aus dem Bewejs von [15: Lemma 4.1 (b)]

Etwas komplizierter gestaltet sich der Fall des Galerkin-Verfahrens. Wenn $\omega(a, \delta)$ den Stetigkeitsmodul. einer Funktion $a \in C(\Gamma)$ bezeichnet, so gilt

$$
\left(a \varphi_{k}{ }^{(n)}, \varphi_{j}^{(n)}\right)=a(\gamma(j / n))\left(\varphi_{k}^{(n)}, \varphi_{j}^{(n)}\right)+O\left(n^{-1} \omega\left(a, n^{-1}\right)\right) .
$$

Da nur $4 d+1$ Diagonalen der Matrix $\left(\left(a \varphi_{k}(n), \varphi_{j}^{(n)}\right)\right)_{j, k=-r}^{r}$ von 0 verschiedene Elemente enthaltén, ergibt sich

$$
\left\|\left(\left(a \varphi_{k}{ }^{(n)}, \dot{\varphi}_{j}^{(n)}\right)\right)_{j, k=-r}^{r}-a_{n} n^{-1} \hat{\alpha}_{n}\right\|_{p}=O\left(n^{-1} \omega\left(a, n^{-1}\right)\right) .
$$

Danit erhält man aus (3.5) für die Matrix $\left.L_{n} a\right|_{s_{n}{ }^{d}}$ beziuglich der Basis $\left\{\varphi_{k}^{(n)}\right\}$ die Beziehungen

$$
\left.L_{n} a\right|_{S_{n^{d}}}=n\left(\alpha^{-1}\right)_{n} \wedge\left(\left(a \varphi_{k}^{(n)}, \varphi_{j}^{(n)}\right)\right)_{i, k}, \quad\left\|\left.L_{n} a\right|_{S_{n}^{d}}-\left(\alpha^{-1}\right)_{n} \wedge a_{n} \hat{\alpha}_{n}\right\|_{p} \rightarrow 0 .
$$

Es sei nun $d=0$ und $a \in P C\left(\Gamma_{0}\right)$. Die Unstetigkeitsstellen von $a$ sollen in der Menge $\left\{\exp \left(i \pi(2 k+1) / n_{0}\right): k=0, \ldots, n_{0}-1\right\}$ für ein gewisses $n_{0} \in \mathbf{X}$. enthalten und $n$ ein ungerades Vielfaches von $n_{0}$ sein. Dann fallen die Sjprungstellen von a unter die Unstetigkeitsstellen der Funktionen aus $S_{n}{ }^{0}$. In diesem Falle läßt sich (4.3) genauso wie für stetige Funktionen $a$ beweisen.

4.2 Der Näherungsoperator der Kollokation für den Cauchyschen singulären Operator besitzt bezüglich der Basis $\left\{\varphi_{n}{ }^{(k)}\right\}$ die Matrix (vgl. (3.7))

$$
K_{n} S_{r_{0}} S_{n_{n}^{a}}=\left(0^{-1}\right)_{n}^{\wedge} \wedge\left(\left(S_{r_{0}} \varphi_{\left.k^{(}\right)}^{(n)}\right)\left(\gamma_{0}((j+\varepsilon) / n)\right)\right)_{j, k=-r}^{r} .
$$


Die Matrixelemente $\left(S_{r_{0} \varphi_{k}}{ }^{(n)}\right)\left(\gamma_{0}((j+\varepsilon) / n)\right)$ lassen sich über die Fourier-Reihe wie folgt bestinmen : '

$$
\left(S_{\Gamma_{0} \varphi_{k}}{ }^{(n)}\right)\left(\gamma_{0}\left(\frac{j+\varepsilon}{n}\right)\right)=\sum_{s \in Z}\left(\varphi_{k}{ }^{(n)}\right)^{\wedge}(s) \operatorname{sign}\left(s_{s}+\frac{1}{2}\right) \exp \frac{i 2 \pi s(j+\varepsilon)}{n}
$$

Unter Verwendung der.Beziehungen (3:2) und (3.3) ergibt sich analog zur Berechnung von $\varphi_{h}^{(n)}(\dot{\gamma}((j+\varepsilon) / n))$ 、

$$
\left(S_{\Gamma_{0} \varphi_{k}}^{(n)}\right)\left(\gamma_{0}\left(\frac{j+\varepsilon}{n}\right)\right)=\frac{1}{. n} \sum_{s=0}^{n-1} \exp \frac{i 2 \pi \dot{s}(j-k)}{n} \sigma\left(\exp \left(i 2 \pi \frac{s}{n}\right)\right)
$$

mit, für $x \in(0,1)$,

$\sigma(\exp (i 2 \pi x)):=\left(I I^{d}\right)^{\wedge}(x) x^{d+1} \exp (i 2 \pi \varepsilon x) \sum_{k \in \boldsymbol{Z}} \frac{\exp (i \pi k(2 \varepsilon+d+1))}{(x+k)^{d+1}} \operatorname{sign}\left(k+\frac{1}{2}\right)$.

Zusammen mit (4.4) erhält man

$$
\left.K_{n} S_{\Gamma_{0}}\right|_{S_{n}}=\left(\varrho^{-1}\right)_{n}{ }^{\wedge} \hat{\sigma}_{n}=\left(\varrho^{-1} \sigma\right)_{n}^{\wedge} .
$$

Lemma 4.2: Die Operaitoren $K_{n} S_{\Gamma_{n}} \mid S_{n}{ }^{a}$.sind in $L^{p}\left(\Gamma_{0}\right)(1<p<\infty)$ gleichmäßig beschränkt, und $\left\{K_{n} S_{\Gamma_{0}} L_{n}\right\}$ konvergiert stark gegen $S_{I_{\bullet}}$.

Beweis: Die Beschränktheit folgt aus (4.5), Lemma 3.1 und Lemma 2.1. Die starke Konvergenz zeigt man wie im Beweis zu [15: L.enma 3.3]

Für die Matrix $L_{n} S_{\Gamma_{0} \mid S_{n}{ }^{a}}$ ergibt sich unter Benutzung von (3.5)

$$
L_{n} S_{\Gamma_{0}} \mid S_{n}^{d}=n\left(\alpha^{-1}\right)_{n} \wedge\left(\left(S_{\Gamma_{0}} \varphi_{k}^{(n)}, \varphi_{j}^{(n)}\right)\right)_{i, k=-r}^{r} .
$$

Das Skalarprodukt $\left(S_{\Gamma_{0}} \varphi_{k}{ }^{(n)}, \varphi_{j}{ }^{(n)}\right)$ wird mit Hilfe der Fourier-Koeffizienten wie folgt bestimmt:

$$
\therefore\left(S_{\Gamma_{0} \varphi_{k}}{ }^{(n)}, \dot{\varphi}_{j}^{(n)}\right)=\sum_{s \in Z}\left(\varphi_{k}^{(n)}\right)^{\wedge}(s) \operatorname{sign}(s+1 / 2) \overline{\left(\varphi_{j}^{(n)}\right)^{\wedge}(s)} .
$$

Anälog zur Berechnung von $\left(\varphi_{k}^{(n)}, \varphi_{j}^{(n)}\right)$ erhält man

$$
\left(S_{\Gamma, \varphi_{k}}^{(n)}, \varphi_{j}^{(n)}\right)=\frac{1}{n^{2}} \sum_{s=0}^{n-1} \exp (i 2 \pi s(j-k) / n) \beta(\exp (i 2 \pi s / n))
$$

nuit, für $x \in(0,1)$,

$$
\beta(\exp (i 2 \pi x)):=\left|\left(\Pi^{d}\right)^{\wedge}(x)\right|^{2} x^{2(d+1)} \sum_{k \in Z}(x+k)^{-2(d+1)} \operatorname{sign}(\dot{k}+1 / 2) .
$$

Zusammen mit (4.6) ergibt sich also

$$
L_{n} S_{\Gamma_{0}} \mid S_{n}{ }^{a}=\left(\alpha^{-1}\right)_{n} \wedge \hat{\beta}_{n}=\left(\alpha^{-1} \beta\right)_{n}^{\wedge} \text {. }
$$

4.3 Nun soll die Matrix des Näherungsoperators für. den singulären Operator $A=a I+b S_{r_{0}}$ bestimmt werden. Aus der für den Interpolationsprojektor bekannten Beziehung $K_{n} b\left(I_{-}-K_{n}\right)=0$, aus (4.1) und (4.5) folgt

$$
\left.K_{n} A\right|_{S_{n}^{d} .}=\left.K_{n} a\right|_{S_{n^{d}}}+\left.\left.K_{n} b\right|_{S_{n}{ }^{d}} K_{n} S_{\Gamma_{i}}\right|_{S_{n^{a}}}=\left(o^{-1}\right)_{n}^{\wedge}\left(a_{n}+b_{n}\left(\varrho^{-1} \sigma\right)_{n}^{\wedge}\right) \hat{\varrho}_{n} .
$$

Für die Orthoprojektoren auf die.Spline-Räume gilt eine zu $K_{n} b\left(I-K_{n}\right)=0$ analoge Beziehung, wie das folgende. Lemma zẹgt. 
Lemma 4.3 (vgl.' [14: Beweis zu Iemma 1.1]): Es sei $f \in C(\Gamma)$ oder $d=0$ und $f \in P C(\Gamma)$, und die Sprungstellen von $f$ mogen zu den Unstetigkeitsstellen der Funktionen aus $S_{n}^{0}$ gehören. Dann gilt $\left\|L_{n} f\left(I-L_{n}\right)\right\| \rightarrow 0$.

Aus diesem Lenma ergibt sich zusammēn mit (4.3) und (4.7)

$$
\left\|\left.L_{n} A\right|_{S_{n} \alpha}-\left(\alpha^{-1}\right)_{n} \wedge\left(a_{n}+b_{n}\left(\alpha^{-1} \beta\right)_{n} \wedge\right) \dot{\alpha}_{n}\right\| \rightarrow 0 \text {. }
$$

Schließlich sei noch bemerkt, daß beim Kollokationsverfahren für den Operator der Gestalt $A=a I+S_{\Gamma_{0}} b$ mit stetigen Koeffizienten eine zu (4.8) analoge Formel gilt. Dies folgt aus

Lemima 4.4 (vgl. [15: Beweis zu Lemma 4.2]): Es sei $d=0$; und f soll einer Lipschitz-Bedingung genügen. Dann gibt es eine Konstante $C>0$ derart, da $\beta$ für alle' $n$ gilt $\| K_{n} S_{\Gamma_{0}}\left(I-K_{n}\right)\left\{L_{n} \| \leqq C(\log n) / n\right.$.

\section{Stabilität spezieller Matrixfolgen mit Zirkulantenstruktur}

5.1 Unı die Stabilität des Kollokationsverfahrens nachzuweisen, genügt es nach Formel (4.8) und Ienma 2.1, die Stabilität der Folge $\left\{a_{n}+b_{n}\left(\varrho^{-1} \sigma\right)_{n}^{\wedge}\right\}$ zu zeigen. $Z u$ diesem Zweck wird in vorliegenden Abschnitt ein Stabilitätskriterium für Matrixfolgen $\left\{B_{n}\right\}$ der Gestalt $B_{n}=a_{n} \hat{\alpha}_{n}+b_{n} \hat{\beta}_{n} \in \mathscr{L}\left(l^{p}(n)\right)$ mit $a, b, \alpha, \beta \in \overline{P C}\left(\Gamma_{0}\right)$ bewiesen. Für dieses Kriterium werglen Operatoren $B_{\varepsilon}$ und $B^{*}$ benötigt, die von $\tau \in \Gamma_{0^{\prime}}$ und $a, b, \alpha, \beta$ abhängen und wie folgt definiert sind:

$$
\begin{aligned}
& B_{\tau}=a\left(\alpha(\tau+0) P_{\Gamma_{0}}+\alpha(\tau-0) Q_{\Gamma_{0}}\right)+b\left(\beta(\tau+0) P_{\Gamma_{0}}+\beta(\tau-0) Q_{\Gamma_{0}}\right) \\
& B^{\tau}=\left(a(\tau+0) P_{\Gamma_{0}}+a(\tau-0) Q_{\Gamma_{0}}\right) \bar{\alpha}+\left(b(\tau+0) P_{\Gamma_{0}}+b(\tau-0) Q_{\Gamma_{0}}\right) \tilde{\beta} .
\end{aligned}
$$

Hierbei wird für $\alpha \in P C\left(\Gamma_{0}\right)$ die Funktion $\tilde{\alpha} \in P C\left(\Gamma_{0}\right)$ durch $\tilde{\alpha}(t)=\alpha\left(t^{-1}\right)$ erklärt.

Lemma 5.1: Für beliebige Funktionen $a, b \in \overline{P C}\left(\Gamma_{0}\right)$ und $\alpha, \beta \in P C^{2}\left(\Gamma_{0}\right)$ ist die Folge $\left\{B_{n}\right\}$ genau dann stabil, wenn für alle $\tau \in \Gamma_{0}$ die Operatoren $B_{r} \in \mathscr{L}\left(L^{p}\left(\Gamma_{0}\right)\right)$ und $B^{\mathrm{r}} \in \mathscr{L}\left(F l^{p}\right)$ invertierbar sind.

Der Beweis dieses Lemmás wird in den Abschnitten 5.2 bis 5.6 geführt werden.

5.2 Zuerst soll gezeigt werden, da $B$ für stabile Folgen $\left\{B_{n}\right\}$ die Operatoren $B_{r}$ aus: $\mathscr{L}\left(L^{p}\left(\Gamma_{0}\right)\right)$ invertierbar sind. Es sei $L_{n}$ der Orthoprojektor aus Abschnitt 3 mit $d=0$. Aufgrund des Lemmas 3.1 kann man die Matrix $B_{n}$ mit dem Operator aus $\mathscr{L}\left(\operatorname{im} L_{n}\right)$ identifizieren, der beziiglich der Basis $\left\{\varphi_{k}^{(n)}\right\}_{k--r}^{r}$ die Matrixdarstellung $B_{n}$ besitzt. Wenn $\left\{B_{n}\right\}$ stabil ist, dann existieren ein $n_{0} \in \mathbf{N}$ und ein $\delta>0$ derart, da $B$ die Ungleichungen

$$
\left\|B_{n} L_{n} f\right\|_{L^{\boldsymbol{D}}\left(\Gamma_{0}\right)} \geqq \delta\left\|L_{n} f\right\|_{L^{p}\left(\Gamma_{0}\right)} \quad\left(f \in L^{p}\left(\Gamma_{0}\right), n \geqq n_{0}\right)
$$

erfiillt sind. Außerdem ist dann die Folge der adjungierten Matrizen $B_{n}^{*} \in \mathscr{L}\left(l^{q}(n)\right.$,) $1 / p+1 / q=1$, stabil, und es gilt analog

$$
\left\|B_{n} * L_{n} g\right\|_{L^{a}\left(\Gamma_{0}\right)} \geqq \delta \hat{\|} L_{n} g \|_{L^{q}\left(\Gamma_{0}\right)}, \quad\left(g \in L^{q}\left(\Gamma_{0}\right), n \geqq n_{0}\right) .
$$

In Lemma 5.2 wird aber gezeigt werden, daß $\left\{B_{n} L_{n}\right\}$ stark gegen $B_{1} \in \mathscr{L}\left(L^{p}\left(\Gamma_{0}\right)\right)$ und $\left\{B_{n}{ }^{*} L_{n}\right\}$ stark gegen $B_{1}^{*} \in \mathscr{I}\left(L^{q}\left(\dot{\Gamma}_{0}\right)\right)$ konvergiert: Durch Grenzübergang in $(\tilde{5} .1)$ und (5.2) erhält mian dann $\left\|B_{1} f\right\|_{L^{p}\left(\Gamma_{0}\right)} \geqq \delta\|f\|_{L^{p}\left(\Gamma_{0}\right)}$ und $\left\|B_{1}{ }^{*} g\right\|_{L^{q^{0}}\left(\Gamma_{0}\right)} \geqq \delta\|g\|_{L^{0}\left(\Gamma_{0}\right)}, \mathrm{d}$. h., $B_{1}$ ist invertierbar. Die Invertierbarkeit von $B_{\tau}$ fïr ein beliebiges $\tau \in \Gamma_{0}$ ergibt sich 
nun folgendermaßen. Man wählt eine Folge von Matrizen $W_{n}{ }^{z} \subset \mathscr{L}\left(l^{p}(n)\right)$ mit $\left\|W_{n}{ }^{r}\right\|_{p}$ $=\left\|\left(W_{n}\right)^{-1}\right\|_{p}=1$ (siehe unten) und zeigt, da $B\left\{W_{n}{ }^{\mathrm{r}} B_{n}\left(W_{n}{ }^{\mathrm{\gamma}}\right)^{-1} L_{n}\right\}$ stark gegen $B_{\tau}$ und $\left\{\left(W_{n}^{\tau} B_{n}\left(W_{n}^{\tau}\right)^{-1}\right)^{*} L_{n}\right\}$ stark gegen $B_{\tau}^{*}$ konvergiert (siehe Lemma 5.2). Wenn nun $\left\{B_{n}\right\}$ stabil ist, so ist auch $\left\{W_{n}^{\tau} B_{n}\left(W_{n}^{\tau}\right)^{-1}\right\}$ stabil, und analog zur Invertierbarkeit von $B_{1}$ schließt man auf die Invertierbarkeit von $B_{\mathrm{r}}$.

Für $\tau=\exp (i 2 \pi s) \in \Gamma_{0}$ und $n \in \mathbf{N}$ sei $k(n, \tau)=\min \left\{k^{\prime} \in \mathbf{Z}: k^{\prime} \geqq n s\right\}$ und ' $W_{n}$ ' $=\left(\exp (-i 2 \pi k(n, \tau) u / n) \delta_{u, v}\right)_{u, v--r}^{r}$. Insbesondere ist $W_{n}{ }^{1}$ die identische Matrix. Wie man leicht zeigen kann, gilt $\left\|W_{n}^{r}\right\|_{p}=\left\|\left(W_{n}^{r}\right)^{-1}\right\|_{p}=1$, und für alle $a, \alpha \in \overline{P C}\left(\Gamma_{0}\right)$ ist

$$
W_{n}^{\gamma} a_{n}=a_{n} W_{n}^{2}, W_{n}^{z} \hat{\alpha}_{n}\left(W_{n}^{2}\right)^{-1}=\left(\dot{\gamma}^{n}\right)_{n}^{\wedge}, \gamma^{n}(t):=\alpha\left(t \exp \frac{i 2 \pi k(n, \tau)}{n}\right) .
$$

Lemma 5.2: Die Folge $\left\{W_{n}{ }^{2} B_{n}\left(W_{n}{ }^{2}\right)^{-1} L_{n}\right\}$ konvergiert stark gegen $B_{\mathrm{r}} \in \mathscr{L}\left(L^{p}\left(\Gamma_{0}\right)\right)$.

Beweis: Aufgrund von (5.3) ist

$$
W_{n} B_{n}\left(W_{n}\right)^{-1}=a_{n}\left(\gamma^{n}\right)_{n}^{\wedge}+b_{n}\left(\lambda^{n}\right)_{n}^{\wedge}, \quad \lambda^{n}(t):=\beta\left(i \exp \frac{i 2 \pi k(n, \tau)}{n}\right) .
$$

Es genügt also, $a_{n} L_{n} \rightarrow a$ und $\left(\gamma^{n}\right)_{n} \wedge L_{n} \rightarrow\left(\alpha(\tau+0) P_{\Gamma_{0}}+\alpha(\tau-0)^{\prime} Q r_{0}\right)$ zu zeigen. Die Konvergenz $a_{n} L_{n} \rightarrow a$ folgt aús Lemma 4.1 und Formel (4.1), bei der in 'Falle $d=0$ die Fuñktion $\varrho$ identisch gleich 1 ist. Die starke Konvergenz von $\left\{\left(\gamma^{n}\right)_{n} \wedge L_{n}\right\}$ zeigt man analog zum Beweis von Lemma 4.2 .

Unter Berücksichtigung von $\left.\left(W_{n}{ }^{\gamma} B_{n}\left(W_{n}{ }^{\gamma}\right)^{-1}\right)^{*}=\overline{\left(\gamma^{n}\right.}\right)_{n}{ }^{\wedge}(\bar{a})_{n}+\left(\overline{\bar{\lambda}^{n}}\right)_{n} \wedge(\bar{b})_{n}$ folgt'aus dem Beweis von. Lemma 5.2 die starke Konvergenz $\left(W_{n}{ }^{r} B_{n}\left(W_{n}\right)^{-1}\right)^{*} L_{n} \rightarrow B_{\varepsilon}{ }^{*}$.

5.3 Analog'zum Abschnitt 5.2 läßt sich auch die Invertierbarkeit der Operatoren $B^{z}\left(\tau \in \Gamma_{0}\right)$ beweisen. Die Rolle des Projektors $L_{n}$ aus Abschnitt 5.2 ibernimmt jetzt der in Teil a) des Beweises vón Lemma 2.1 eingeführte Projektor $P_{n}$, der aber

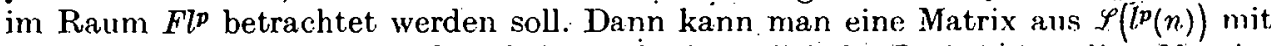
den Operator aus $\mathscr{L}\left(\operatorname{im} P_{n}\right)$ identifizieren, der bezüglich der Basis $\left\{\boldsymbol{i}^{i}\right\}_{j--r}^{r}$ diese Matrixdarstellung besitzt. An Stelle von $W_{n}$ benötigt man Matrizen $V_{n}{ }^{z}$, die wie folgt definiert werden: Für $\tau=\exp (i 2 \pi s) \epsilon \Gamma_{\mathbf{0}}$ und $n \in \mathbf{N}$ sei $m(n, \tau)=\min \left\{m^{\prime} \in \mathbf{Z}: m^{\prime}\right.$ $\geqq n s-\varepsilon\} \quad$ (vgl. (4.1)) und $V_{n}{ }^{2}:=U^{1}\left(\exp (-i 2 \pi m(n, \tau) u / n) \delta_{u, v}\right)_{u, v--,}^{r} U^{\prime} \quad(\mathrm{vgl}$. Abschnitt 2.1). Wie man leicht zeigen kann, gilt $\left\|V_{n}^{r}\right\|_{p}=\left\|\left(V_{n}^{\prime}\right)^{-1}\right\|_{p}=1$ (vgl. Youngsche Ungleichung), und für alle $a, \alpha \in \overline{P C}\left(\Gamma_{0}\right)$ ist

$$
V_{n}^{\mathrm{r}} \hat{\alpha}_{n t} \doteq \hat{\alpha}_{n} V_{n}^{\mathrm{\gamma}}, V_{n}^{\mathrm{r}} a_{n}\left(V_{n^{\mathrm{r}}}\right)^{-1}=\left(c^{n}\right)_{n}, \quad, c^{n}(t):=a\left(t \exp \frac{i 2 \pi m(n, \tau)}{n}\right) .
$$

Lemma 5.3: Die Folge $\left\{V_{n}{ }^{\mathrm{r}} B_{n}\left(V_{n}\right)^{-1} P_{\dot{n}}\right\}$ konvergiert slark gegen $B^{\mathrm{r}} \in \mathscr{L}\left(F l^{p}\right)$.

Beweis: Aufgrund von (5.4) ist

$$
V_{n} B_{n}\left(V_{n}{ }^{2}\right)^{-1}=\left(c^{n}\right)_{n} \hat{\alpha}_{n}+\left(d^{n}\right)_{n} \hat{\beta}_{n}, \quad d^{n}(t) \vdots=b\left(t \exp \frac{i 2 \pi m(n, \tau)}{n .}\right) .
$$

Es genügt also, $\left(c^{n}\right)_{n} P_{n} \rightarrow\left(c(\dot{\tau}+0) P_{\Gamma_{0}}+c\left(\tau^{-}-0\right) Q_{\Gamma_{0}}\right)$ und $\hat{\alpha}_{n} P_{n}^{-} \rightarrow \tilde{\alpha}$ zu zeigen. Die Konvergenz von $\left\{\left(c^{n}\right)_{n} P_{n}\right\}$ ist aufgrund der Diagonalgestalt der Operatoren bezüglich der Basis $\left\{\boldsymbol{t}^{j}\right\}_{j \in Z}$ offensichtlich. Für die Konvergenz von $\left\{\hat{\alpha}_{n} P_{n}\right\}$ führt man den Interpolationsprojektor $M_{n} \cdot$ ein, der für alle Riemann-integrierbaren Funktionen $f$ durch

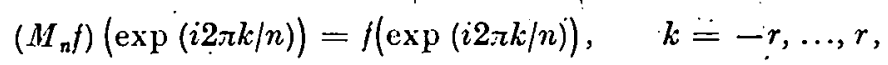


sowie $M_{n} f \in$ im $P_{n}$ erklärt wird (vgl. [23]). Dann hat der Näherungsoperator $\left.M_{n} \tilde{\alpha}\right|_{1 \mathrm{~m} P_{n}}$, der bei der trigonometrischen Kollokation für den Multiplikationsoperator $\bar{\alpha}$ entsteht, bezüglich der Basis $\left\{t^{j}\right\}_{j--}^{r}$ die Matrixdarstellung $\hat{\alpha}_{n}$. Aus [11: Hilfssatz 2.4] folgt die $F l^{2}$-Konvergenz $\hat{\alpha}_{n} P_{n} \rightarrow \tilde{\alpha}$. Unter Ausnutzung der gleichmäBigen Beschränktheit von $\left\{\hat{\alpha}_{n} P_{n}\right\}$ und der Hölderschen Ungleichung ergibt sich die Konvergenz in $F^{p}(1<p<\infty)$

Die Matrix $\left(V_{n}^{r} B_{n}\left(V_{n}\right)^{-1}\right)^{*}$ hat die Gestalt $(\bar{\alpha})_{n} \wedge\left(\overline{c^{n}}\right)_{n}+(\bar{\beta})_{n} \wedge\left(\overline{d^{n}}\right)_{n}$. Nach Lemma 5.3 gilt also auch $\left(V_{n}^{3} B_{n}\left(V_{n}^{r}\right)^{-1}\right)^{*} P_{n} \rightarrow\left(B^{\mathrm{r}}\right)^{*} \in \mathscr{L}\left(F^{\prime} l^{q}\right)(1 / p+1 / q=1)$. Hierńit und unter Benutzung von Lemma 5.3 zeigt man analog zum A Abschnitt 5.2 die Invierticrbarkeit von $B^{\mathrm{r}} \in \mathscr{L}\left(F^{l} l^{p}\right)$.

5.4 Die Hinlänglichkeit der in Lemma $\tilde{\mathbf{5}} .1$ formulierten Stabilitätsbedingungen ergibt sich aus einem lokalen Prinzip für. Näherungsverfahren (siehe nachfolgendes Jemma 5.4). Der vollständige Beweis dieses Prinzips ist in [17] angegeben. Er beruht auf den Beweisgedanken zu den lokalen Prinzipien aus $[9,11]$.

Der Operator $L_{n}(d=0)$ ' soll wie im Abschnitt 3.2 definiert sein. Ähnlich wie in Abschnitt 5.2 werden im weiteren die Räume $\mathscr{L}\left(l^{p}(n)\right)$ und $\mathscr{L}\left(\operatorname{im}, L_{n}\right)$ identifiziert. Dá nach'Lenma 5.2 die Folge $\left\{B_{n} L_{n}\right\}$ stark gegen $B_{1}$ konvergiert, kann man für den Operator $B_{1} \in \mathscr{L}\left(L^{p}\left(\dot{\Gamma}_{0}\right)\right)$ das Näherungsverfahren mit den Operatoren $B_{n}$ betrachten. Um die Stabilitäl dieses Verfahrons $z u$ beweisen, treffen wir die folgenden Vereinharungen: Die Menge aller Folgen $\left\{m_{n}\right\}_{n \in \mathbb{N}}$, wobei $m$ eine auf $\Gamma_{0}$ beliebig oft differenzierbare nichtnegative Funktion ist, sei mit $\mathfrak{M}$ bezeichnet. Ferner sei $\left\{\tau_{0}=-1, \tau_{1}, \ldots\right.$, $\left.\tau_{R}\right\}(R \in \mathbf{N})$ eine fixierte. Teilmenge von $\Gamma_{0}$, die alle Sprungstellen der Funktionen $\alpha$ und $\beta$ enthält, und $W_{n}^{i}=W_{n}{ }^{r_{i}}$ für $i=0,1, \ldots, R$. Schließlich sei $\Re$ die Menge aller Linearkombinationen von Matrixfolgen der Form $\left\{b_{n}\left(W_{n}^{i}\right)^{-1} L_{n} T W_{n}{ }^{i} D_{n}+C_{n}\right\}$, wobei $b \in \overline{P C}\left(\Gamma_{0}\right)$ ist, $T \in \mathscr{L}\left(L^{p}\left(\Gamma_{0}\right)\right)$ ein kompakter Operator und $C_{n}, D_{n} \in \mathscr{L}\left(l^{p}(n)\right)$ Operatoren mit sup $\left\{\left\|D_{n}\right\|_{p}: n \in \mathbf{N}\right\}<\infty$,bzw. $\left\|C_{n}\right\|_{p} \rightarrow 0$ sind. Mit diesen Bezeichnungen sollen drei Voraussetzungen erfüllt sein :

a) Es sollen invertierbare Operatoren $B^{i} \in \mathscr{L}\left(L^{p}\left(\Gamma_{0}\right)\right)$ derart existieren, da $\beta$ $\left\{W_{n}^{i} B_{n}\left(W_{n}^{i}\right)^{-1} L_{n}\right\}$ stark gegen $B^{i}$ konvergiert.

b) Für alle $\left\{m_{n}\right\} \in \mathfrak{N}$ gelte $\left\{m_{n} B_{n}-B_{n} m_{n}\right\} \in \mathfrak{N}$.

c) Für ein gewisses $N \in \mathbf{N}$ sollen Matrizenfolgen $\left\{m_{n}{ }^{j}\right\} \in \mathfrak{M}$ und $\left\{R_{n}{ }^{j}\right\}(j=1, \ldots, N)$ derart existieren, daß sup $\left\{\left\|R_{n}{ }^{j}\right\|_{p}: n \in \mathbf{N}\right\}<\infty$ und $m_{n}{ }^{i} B_{n} R_{n}{ }^{j}=m_{n}{ }^{j}$ ist. Die Summe $f_{n}=m_{n}{ }^{\mathbf{1}}+\cdots+m_{n}{ }^{N}$ soll für genügend großes $n$ invertierbar und die Folge der inversen Matrizen $\left\{f_{n}^{-1}\right\}$ gleichmäßig beschränkt sein.

Lemma 5.4 (siche [17]): Wenn die Voraussetzungen a)-c) erfüllt sind, dann ist die Folge $\left\{B_{n}\right\}$ stabil.

Für den Beweis der Hinlänglichkeit der Stabilitätsbedingungen aus Lemma 5.1 genügt es also, die Giiltigkeit, der Bedingungen a)-c) zu zeigen.

5.5 Falls die Stabilitätsbedingungen aus Lemma 5.1 erfüllt sind, folgt die Bedingung a) aus Lcmma 5.2. Die Bedingung b) folgt aus $m_{n} a_{n}=a_{n} m_{n}, m_{n} b_{n}=b_{n} m_{n}$ únd dem folgenden

Lem mे 5.5: (i) Aus $\left\{m_{n}\right\} \in$ Mg und $\alpha \in C\left(\Gamma_{0}\right) \cap P C^{2}\left(\Gamma_{0}\right)$ folgt $\left\|m_{n} \hat{\alpha}_{n}-\hat{\alpha}_{n} m_{n}\right\|_{p} \rightarrow 0$.

(ii) Aus $\left.\left\{\dot{m}_{n}\right\} \in \mathbb{N}\right\}$ und $\underset{n}{\alpha} \in P C^{2}\left(\Gamma_{0}\right)$ folgt $\left\{m_{n} \hat{\alpha}_{n}-\dot{\alpha_{n}} m_{n}\right\} \in \mathfrak{\Omega}$.

Beweis: (i) Aus (4.2) und $|\hat{\alpha}(k)| \leqq C k^{-2}$ folgt für eine geeignete (andere) Konstante $C>0$ und für alle $k \in\{-r, \ldots, r\}$ die Abschätzung $\left|\hat{\alpha}_{n, k}\right| \leqq C k^{-\hat{2}}+C n^{-2}$. Zusammen mit $\left|m\left(\gamma_{0}((k+\varepsilon) / n)\right)-m\left(\gamma_{0}((l+\varepsilon) / n)\right)\right| \leqq C(k \dot{-l}) / n$ ergibt sich für 
das Element der $k$-ten Zeile und $l$-ten Spalte von $\left(\hat{m_{n} \hat{\alpha}_{n}}-\hat{\alpha}_{n} m_{n}\right)$ die Abschätzung

$$
\begin{array}{r}
\left|\left(m\left(\gamma_{0}((k+\varepsilon) / n)\right)-m\left(\gamma_{0}((l+\varepsilon) / n)\right)\right) \hat{\alpha}_{n, k<\iota}\right| \leqq C(k-l)^{-1} n^{-1} \\
+C\left(k \dot{-l)} n^{-3} .\right.
\end{array}
$$

Unter Benutzung der Youngschen Ungleichung schließt man

$$
\begin{aligned}
\left\|m_{n} \hat{\alpha}_{n}-\hat{\alpha}_{n} m_{n}\right\|_{p} & \leqq C\left\|\left(\left((k-l)^{-1} n^{-1}+(k-l) n^{-3}\right)\right)_{k, l--r}^{r}\right\|_{p} \\
& \leqq C n^{-1} \log n \rightarrow 0 .
\end{aligned}
$$

(ii) Es sei $\dot{x}:=\varrho^{-1} \sigma\left(\operatorname{vgl}\right.$. (4.5)), und für $t \in \epsilon \Gamma_{0}$ und alle $s \in \Gamma_{0}$ sei $x^{t}(s):=\varkappa\left(t^{-1} s\right)$. Dann ist $\alpha^{\prime}:=\alpha-\sum_{j=0}^{R} 1 / 2\left(\alpha\left(\tau_{j}+0\right)-\alpha\left(\tau_{j}-0\right)\right) \varkappa^{r j} \in P C^{2}\left(\Gamma_{0}\right) \cap C\left(\Gamma_{0}\right)$, denn es gilt $x(1 \pm 0)= \pm 1$. Also genügt es, $\left\{m_{n}\left(x^{\mathrm{r}}\right){ }_{n}{ }^{\wedge}-\left(\varkappa^{\mathrm{r}}\right)_{n} \wedge m_{n}\right\} \in \mathfrak{S i}$ zu zeigen. Wenn $\varkappa^{n}$ $(n \in \mathbf{N})$ durch $x^{n}(s)=x\left(\exp \left(-i 2 \pi k\left(n, \tau_{j}\right) / n\right) s\right)$ erklärt wird, konvergiert ' $\|\left(x^{i}\right)_{n}$ - $\left(\varkappa^{n}\right)_{n} \|_{2}$ aufgrund der Definition von $k\left(n, \tau_{j}\right)$ gegen 0 . Weil die Fourier-Transformation $U \in \mathscr{L}\left(l^{2}(n)\right)$ isometriśch ist, ergibt sich unter Benutzung von (5.3) dic Konvergenz $\left\|\left(x^{\tau^{j}}\right)_{n} \wedge-\left(W_{n}^{j}\right)^{-1} \hat{x}_{n} W_{n}^{j}\right\|_{2} \rightarrow 0$. Diese Nullfölge ist in $\mathscr{L}\left(l^{p}(n)\right)(1<p<\infty)$ bezüglich $n$ gleichmäßig beschränkt. Aus der Interpolationstheorie (siehe [21]) folgt $\left\|\left(*^{\tau_{j}}\right)_{n}^{\wedge}-\left(W_{n}^{j}\right)^{-1} \hat{\varkappa}_{n} W_{n}^{j}\right\|_{p} \rightarrow 0$. Es '́genïgt also,

$$
\left\{m_{n}\left(W_{n}^{j}\right)^{-1} \hat{x}_{n} W_{n}^{j}-\left(W_{n}^{j}\right)^{-1} \hat{\varkappa}_{n} W_{n}^{j} m_{n}\right\} \in \mathfrak{N},
$$

d. h. $\left\{\left(W_{n}^{j}\right)^{-1}\left(m_{n} \hat{\varkappa}_{n}-\hat{x}_{n} m_{n}\right) W_{n}^{j}\right\} \in \mathfrak{N}$

zu zeigen. Die Formèln (4.1) und (4.5) ergeben $m_{n} \hat{x}_{n}-\hat{\varkappa}_{n} m_{n}=K_{n}{ }_{n} K_{n} S_{r_{0}} L_{n}$ $-K_{n} S_{\Gamma_{0}} K_{n} m L_{n}$. Dann muB wegen $K_{n} m\left(l-K_{n}\right)=0$ und Lemma 4.4 nur noch $\left\{\left(W_{n}{ }^{j}\right)^{-1} K_{n}\left(m S_{\Gamma_{0}}-S_{\Gamma_{0}} m\right) W_{n}{ }^{j}\right\} \in \Re$ bewiesen werden. Da $m S_{\Gamma_{0}}-S_{r_{0}} m$ den Raum $L^{p}\left(\Gamma_{0}\right)$ kompakt in $C\left(\Gamma_{0}\right)$ abbildet und die Folgen $\left\{K_{n}\right\}$ und $\left\{L_{n}\right\}$ stark gegen die Einbettung von $C\left(\Gamma_{0}\right)$ nach $L^{p}\left(\Gamma_{0}\right)$ konvergieren (vgl. Lemma 3.3 und Lemma 3.2 ), ist $\left\{\left(K_{n}-L_{n}\right)\left(m S_{\Gamma_{0}}-S_{\Gamma_{0}} m\right)\right\}$ eine Nullfolge des Raumes $\mathscr{L}\left(L^{p}\left(\Gamma_{0}\right)\right)$. Aus $\left\{\left(W_{n}^{j}\right)^{-1} L_{n}\left(m S_{\Gamma_{0}}\right.\right.$ $\left.\left.-S_{I_{0}} m\right) W_{n}{ }^{j}\right\} \in \Re$ folgt die Behauptung

5.6 Jetzt wird bewiesen, da $B$ zu jedem $\tau \in \Gamma_{0}$ Folgen $\left\{m_{n}{ }^{x}\right\} \in \mathbb{M}$ und $\left\{R_{n}{ }^{x}\right\}$ existieren, so da $B m^{x}(\tau)=1$ und $m_{n}{ }^{\gamma} B_{n} R_{n}{ }^{x}=m_{n}{ }^{x}$ gilt. Ist dies gezeigt, dann kann man Punkte $\tau_{i} \in \Gamma_{0}(i=1, \ldots, N)$ mit $f=m^{\tau_{1}}+\cdots+m^{t_{N}}>0$ wählen, und die Bedingung $\left.c\right)$ ist für $m_{n}{ }^{i}=\grave{m}_{n}{ }^{{ }^{\prime}}$ und $R_{n}{ }^{i}=R_{n}{ }^{{ }^{i}}$ erfüllt.

Wie im Abschnitt.5.3 soll wieder $\mathscr{L}\left(l^{p}(n)\right)$ mit $\mathscr{L}\left(\right.$ im $\left.P_{n}\right)$ identifiziert werden. Für alle $\left\{m_{n}\right\},\left\{m_{n}{ }^{\prime}\right\},\left\{m_{n}{ }^{\prime \prime}\right\}$ mit $m_{n} m_{n}^{\prime \prime}=m_{n}, m_{n}{ }^{\prime \prime} m_{n}{ }^{\prime}=m_{n}^{\prime \prime}$ und $m(\tau)=1$ gilt

$$
m_{n} B_{n} m_{n}{ }^{\prime}\left(V_{n}\right)^{-1}=m_{n}\left(P_{n} B^{\mathrm{r}}+D_{n}^{1}+\cdots+D_{n}^{6}\right)
$$

mit

$$
\begin{aligned}
& D_{n}{ }^{1}=\left(V_{n}\right)^{-1}\left[V_{n}{ }^{\mathrm{z}} a_{n}\left(V_{n}^{\mathrm{s}}\right)^{-1}-\left(a(\tau+0) P_{r_{0}}+a(\tau-0) Q_{\Gamma_{0}}\right)\right] \\
& \times V_{n}^{\mathrm{r}} m_{n}{ }^{\prime}\left(V_{n}^{\mathrm{r}}\right)^{-1} \hat{\hat{\alpha}}_{n} V_{n}^{\mathrm{s}} m_{n}{ }^{\prime}\left(V_{n}^{\mathrm{r}}\right)^{-1} \text {, } \\
& D_{n}^{2}=\left(V_{n}^{\tau}\right)^{-1}\left[V_{n}^{\mathrm{z}} b_{n}\left(V_{n}^{\mathrm{r}}\right)^{-1}-\left(b(\tau+0) P_{r_{0}}+b(\tau-0) Q r_{0}\right)\right] \\
& \times V_{n}^{\mathrm{z}} m_{n}^{\prime \prime}\left(V_{n}^{\mathrm{z}}\right)^{-1} \hat{\beta}_{n} V_{n}^{\mathrm{t}} m_{n}^{\prime}\left(V_{n}^{\mathrm{t}}\right)^{-1} \text {, }
\end{aligned}
$$

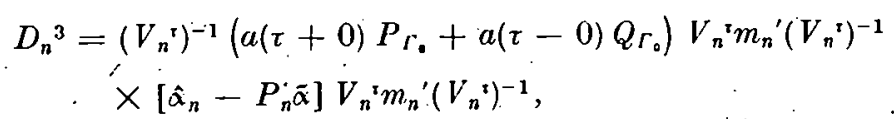



Also gilt $\hat{\alpha}_{n, l, k}-\hat{\alpha}_{n, l}=\hat{\alpha}_{n, l, k}^{1}+\hat{\alpha}_{n, l, k}^{2}$ mit

$$
\begin{aligned}
& \hat{\alpha}_{n, l, k}^{1}=\sum_{s=-k n+1}^{k n} \frac{\exp (i 2 \pi l s / 2 k n)}{2 k n}\left[\alpha(\exp (i 2 \pi s / 2 k n))-\alpha\left(\exp \left(i 2 \pi s^{\#} / 2 k n\right)\right)\right], \\
& \dot{\alpha}_{n, l, k}^{2}=\sum_{s=-k n+1}^{k n} \frac{\alpha\left(\operatorname { e x p } \left(i 2 \pi s^{\#, / 2 k n n))}\right.\right.}{2 k n}\left[\exp (i 2 \pi s l / 2 k n)-\exp \left(i 2 \pi l s^{\#} / 2 k n\right)\right] .
\end{aligned}
$$

Wenn'zwischen $\exp (i 2 \pi s / 2 k n)$ und $\exp \left(i 2 \pi s^{\#} / 2 k n\right)$ keine Sprlıngstelle von $\alpha$ liegt, gilt $\left|\alpha(\exp (i 2 \pi s / 2 k n))-\alpha\left(\exp \left(i 2 \pi s^{\#} / 2 k n\right)\right)\right|<C n^{-1}$. Somit ergibt. sich $\left|\hat{\alpha}_{n, l, k}^{1}\right|<C n^{-1}$. Für $\hat{\alpha}_{n, l, k}^{2}$ erhält man

$$
\hat{\alpha}_{n, l, k}^{\dot{2}}=\hat{\alpha}_{n, l} \beta_{n, l, k} \quad \text { mit } \quad \beta_{n, l, k}=\frac{1}{2 k} \sum_{u=-k+1}^{k}[\exp (i 2 \pi l u / 2 k n)-1]
$$

Mit Hilfe dẹ Summenformel.für die geometrische Reihe schließt mian

$$
\beta_{n, l . k}=\frac{\sin (\pi l / n)}{\pi l / n} \frac{i 2 \pi l / 2 k n}{\exp (i 2 \pi l / 2 k n)-1} \exp (i 2 \pi l / 2 k n)-1
$$

und

$$
\operatorname{limi}_{k \rightarrow \infty} \beta_{n, l, k}=\frac{\sin (\pi l / n)}{\pi l / n}-1=(l / n)\left\{\pi \sum_{u=1}^{\infty} \frac{1}{(2 u+1) !}(\pi l / n)^{2 u-1}\right\} .
$$

Folglich gilt für $(\tilde{\alpha})^{\wedge}(l)-\hat{\alpha}_{n, l}=\lim _{k \rightarrow \infty}\left(\hat{\alpha}_{n, l, k}-\hat{\alpha}_{n, l}\right)$ die Abschätzung $\mid(\tilde{\alpha})^{\wedge}(l)-\hat{\alpha}_{n, l} !^{\prime}$ $\leqq C n^{-1}+C\left|\hat{\alpha}_{n, l}\right| l n^{-1}$. Zusammen mit $\left|(\bar{\alpha})^{\wedge}(l)\right| \leqq C l^{-1}$ crgibt sich $\left|(\bar{\alpha})^{\wedge}(l)-\hat{\alpha}_{n . l}\right|$ $\leqq C n^{-1}+C\left|(\tilde{\alpha})^{\wedge}(l)-\hat{a}_{n, l}\right| l n^{-1}$, woraus unmittelbar (5.6) folgt.

b) Wenn die Umgebung $U$ in Lemma 5.8 genügend klein gewählt wird, dann enthält die Matrix $\left\{m_{n}\left(\hat{\alpha}_{n}-P_{n} \tilde{\alpha}\right) m_{n}\right\}$, abgesehen von einer $[\delta n] \times[\delta n]$-Untermatrix, nur Nullelemente. Die von 0 verschiedenen Elemente dieser Matrix sind aufgrund von a) kleiner oder gleich $\mathrm{Cn}^{-1}$. Aus der Youngschen Ungleichung folgt aber, da $B$ die Norm einer solchen Matrix kleiner oder gleich $2 C \delta$ ist

\section{Stabilität spezicller Näherungsverfahren}

6.1 Es soll zuerst das $\varepsilon$-Kollokationsverfahren mit Spline-Ansatzfunktionen für $\varepsilon \in(-1 / 2,1 / 2)$ betrachtet werden. Dazu sei $\varkappa$ die Funktion $\varrho^{-1} \sigma$ aus (4.5), d. h., für $x \in(0,1)$ sei

$$
x(\exp (i 2 \pi x)):=\frac{\sum_{k \in \mathbf{Z}} \exp (i \pi k(2 \varepsilon+d+1))(x+k)^{-d-1} \operatorname{sign}(\dot{k}+1 / 2)}{1-\sum_{k \in \mathbf{Z}} \exp (i \pi k(2 \varepsilon+d+1))(x+k)^{-d-1}} .
$$

Satz 6.1: Für $\varepsilon \in(-1 / 2,1 / 2), \quad a, b \in \overline{P C}\left(\Gamma^{\prime}\right)$ und $\dot{A}=a I+b S_{\Gamma} \in \mathscr{L}^{p}\left(L^{p}(\Gamma)\right)$ $(1<p<\infty)$ ist die $\varepsilon$-Kollokation genau dann stabil, wenn der Operator $A \in \mathscr{L}\left(L^{p}(F)\right)$ und für alle $\tau \in \Gamma$ die Operatoren

$$
A^{z}:=P_{\Gamma_{0}}(a(\tau+0)+b(\dot{\tau}+0) \tilde{x})+Q_{\Gamma_{0}}(a(\tau-0)+b(\tau-0) \tilde{x}) \in \mathscr{L}\left(F l^{p}\right)
$$

invertierbar sind. 
Beweis: a) Zuerst sei $\Gamma=\Gamma_{0}$. Nach Abschnitt 5.1 ist die $\varepsilon$-Kollokation genau dann stabil, wenn die Folge $\left\{a_{n}+b_{n} \hat{x}_{n}\right\}$ stabil ist. Aus Lemma 5.1 folgt die Behauptung.

b) Es sei $\gamma$ die.Parameterdarstellung einer beliebigen Kurve $\Gamma$. Wenn $\left\{\varphi_{k}^{(n)}\right\}$ die in Abschnitt 3.1 definierte Basis des Raumes $S_{n}^{d}$ auf $\Gamma$ und $\left\{\varphi_{0, k}^{(n)}\right\}$ die entsprechende Basis des Spline-Raumes $S_{0, n}^{d}$ a uf $\Gamma_{0}$ bezeichnen, dann gilt $\varphi_{k}{ }^{(n)}=\varphi_{0, k}^{n} \circ \chi^{-1}(k \doteq 0, \ldots$, $\left.n-1 ; \chi:=\gamma \circ \gamma_{0}^{-1}\right)$. Es sei $K_{n}$ der in Abschnitt 3.3 eingeführte Interpolationsprojektor auf $\Gamma$ und $K_{0, n}$ der entsprechende Projektor-auf $\Gamma_{0}$. Ferner sei $a_{0}:=a \circ \%$ $b_{0}:=b_{.} \circ \chi, A_{0}:=a_{0} I+b_{0} S_{\Gamma_{0}}$ und für $x \in L^{p}\left(\Gamma_{0}\right)$ der Operator $T$ durch

$$
\text { . } \quad(T x)(l)=b_{0}(l) \frac{1}{\pi i} \int_{\Gamma_{0}} x(s)\left\{\chi^{\prime}(s)\left(\chi(s)^{i}-\chi(t)\right)^{-1}-(s-t)^{-1}\right\} d s
$$

definiert. Offensichtlich besitzt der Kollokationsoperator $\left.K_{n} A\right|_{S_{n}{ }^{a}}$ bezüglich $\left\{\dot{\varphi}_{k}^{(n)}\right\}_{k=0}^{n-1}$ die gleiché Matrix wie $\left.K_{0, n}\left(A_{0}+T\right)\right|_{S_{0, n}^{d}}$ bezüglich der Basis $\left\{\varphi_{0, k}^{(n)}\right\}_{k=0}^{n-1}$. Aus Lenima 3.1 folgt, da $\dot{B}$ die $\varepsilon$-Kollokation für den Operator $A$ geriau dann stabil ist, wenn sie fuir $A_{0}+T$ stabil ist. Der Operator $T$ bildet aber $L^{p}\left(\Gamma_{0}\right)$ kompakt in $\overline{P C}\left(\Gamma_{0}\right)$ ab. Unter Benutzung bekannter Störungssätze (vgl. $[8,16])$ ergibt sich, daß die $\varepsilon$-Kollokation für $A_{0}+T$ genau dann stabil ist, wenn sie für $A_{0}$ stabil ist. Damit ist der Fall einer beliebigen Kurve $\Gamma$ auf den Spezialfall $\Gamma=\Gamma_{0}$ zurückgeführt

Bemerkung 6.1: Für $\Gamma^{\top}=\Gamma_{0}$ folgt aus Lemma 4.1 und Lemma 4.2 die Konvergenz $K_{n} A L_{n} \rightarrow A$. Analog zum Teil b) des vorstehenden Beweises läßt sich diese Konvergenz für eine beliebige Kurve $\Gamma$ zeigen. Wenn die $\varepsilon$-Kollokation stabil ist und wenn die rechte Seite $y$ Riemann-integrierbar ist, dann bonvergieren die Näherungslösungen $x_{n}$ des Kollokationsverfahrens gegen die exakte Lösung der Gleichung (vgl. Lemma 3.3 und Abschnitt 1.1).

Folgerung 6.1: Für $a, b \in \overline{P C}(\Gamma)$ und $A=a I+b S_{\Gamma} \in \mathscr{L}\left(L^{p}(\Gamma)\right)$ ist die 0-Kollokalion genau dann stabil, wenn für alle $t \in \Gamma$ entweder die Halbgerade $(-\infty ; 0]$ den Kreisbogen $K$ aus $(0.2)$ nicht schneidet oder $(-\infty, 0)$ den Bogen $K$ in zwei inneren Punkten schneidet.

Beweis: Es sei $v=\frac{1}{2}(1+\tilde{x})$ und

$$
K_{1}=\left\{\left[d^{-1} c(t+0) \mu \nmid(1-\mu)\right] /\left[\mu+c^{-1} d(t-0)(1-\mu)\right]: \mu \in[0,1]\right\} .
$$

Der Operator $A^{t}$ ist offensichtlich genau dann invertierbar, wenn

$$
\begin{aligned}
& \left(P_{r_{0}} c(t-0) / d(t+0)+Q_{r_{0}}\right) A^{t}(a(t-0)+b(t-0) \bar{x})^{-1} \\
& \left.=\dot{P}_{r_{0}}\left\{v d^{-1} c(t+0)+(1-v)\right] /\left[v+(1-v) c^{-1} d(t-0)\right]\right\}+Q_{r_{0}}
\end{aligned}
$$

in Raum $F l^{p}$ invertierbar ist. Aufgrund des Invertierbarkeitskriteriums aus $A$ bschnitt 1.2 ist dies dann und nur dann der Fall, wenn der Koordinatenursprung nicht in das von $K_{1}$ und $K$ eingeschlossene Gebiet $G$ fällt. Aus der fiir alle $\nu$ und $\mu \in[0,1]$ gïltigen Äquivalenz der Beziehungen

und

$$
v d^{-1} c(\ell+0)+(1-u) d^{-1} c(l-0)=-\mu^{-1}(1-\mu)
$$

$$
\begin{aligned}
& v\left[d^{-1} c(t+0) \mu+(1-\mu)\right] /\left[\mu_{1}+(1-\mu) c^{-1} \dot{d}(t-0)\right] \\
& +(1-v) d^{-1} c(t-0)=0
\end{aligned}
$$

folgt, daß $(-\infty, 0]$ die Strecke $S^{\prime}=\left\{\mu d^{-1} c(t+0)+(1-\mu) d^{-1} c(t-0): \mu \in[0,1]\right\}$ genau dann schneidet, wenn 0 zu dem von $S$ und $K_{1}$ eingeschlossenen Gebiet gehört. 
Falls $(-\infty, 0]$ die Strecke $\$$ schneidet, liegt 0 dann und nur dann in $G$, wenn $(-\infty, 0]$ den Bogen $K$ in einem inneren Punkt schneidet. Falls $(-\infty, 0]$ die Strecke $S$ nicht schneidet, liegt 0 wiederum genau dann in $G$, wenn $(-\infty, 0]$ den Bogen $K$ in einem inneren. Punkt schneidet

In Falle $\varepsilon=1 / 2$ existiert kein Interpolationsprojektor mit dem Bildraum $S_{n}{ }^{d}$. Es sei deshalb $\left(S_{n}{ }^{d}\right)^{\prime}$ der in der Pa rameterdarstellung um $(2 n)^{-1}$ verschobene Spline-Raum, d. h. $\psi$ gehört zu $\left(S_{n}{ }^{d}\right)^{\prime}$ genau dann, wenn ein $\varphi \in S_{n}{ }^{d}$ mit $\psi(\gamma(s))=\varphi\left(\gamma\left(s+(2 n)^{-1}\right)\right)$ für alle $s \in \mathbf{R}$ existiert. Wenn $K_{n}{ }^{\prime}$ den durch $K_{n}{ }^{\prime} f \in\left(S_{n}{ }^{d}\right)^{\prime}$ und $\left(K_{n}{ }^{\prime} f\right)(\gamma((k+1 / 2) / n))$ $=f(\gamma((k+1 / 2) / n))(k=-r, \ldots, r)$ definierten Interpolationsprojektor bezeichnet, dann ist das Näherungssystem der $1 / 2$-Kollokation äquivalent zu $K_{n}^{\prime} A x_{n}=K_{n}^{\prime \prime} y$. Analog wie in Abschnitt 1.1 erklärt man die Stabilität der Operatoren $\left.K_{n}{ }^{\prime} A\right|_{S_{n}{ }^{d}}$ : $S_{n}{ }^{d} \rightarrow\left(S_{n}{ }^{d}\right)^{\prime}$.

Satz 6.2: Für $a, b \in \overline{P C}(\Gamma)$ und $A=a I+b S_{l} \in \mathscr{L}\left(L^{p}(\Gamma)\right)(1<p<\infty)$ ist die 1/2-Kollokation genau dann stabil, wenn für alle $t \in \Gamma$ entweder die Halbgerade $[0, \infty)$. den Kreisbogen $K$ aus (0.2) nicht schneidet oder $(0, \infty)$, den Bogen $K$ in zwei inneren Punkten schneidet.

Beweis: Das durch $\left(\varphi_{k}^{(n)}\right)^{\prime}(\gamma(s))=\varphi_{k}^{(n)}\left(\gamma\left(s+(2 n)^{-1}\right)\right)$ definierte Funktionssystem $\left\{\left(\dot{\varphi}_{k}{ }^{(n)}\right)^{\prime}\right\}_{k=0}^{n-1}$ ist eine Basis in $\left(S_{n}{ }^{d}\right)^{\prime}$. Analog $z u(4.8)$ zeigt man, daß die Matrix von $\left.K_{n}{ }^{\prime} A\right|_{S_{n}{ }^{d}}$ bezüglich der Basen $\left\{\varphi_{k}^{(n)}\right\}$ und $\left\{\left(\varphi_{k}^{(n)}\right)^{\prime}\right\}$ die Gestalt $\left(g^{\prime \cdots 1}\right)_{n}^{\wedge}\left\{a_{n}\left(\varrho \sigma^{1}\right)_{n} \wedge+b_{n}\right\} \hat{\sigma}_{n}$ besitzt, wobei $\varrho$ und $\sigma$ die gleiche Bedentung wie in Abschnitt $4(\varepsilon=1 / 2)$ haben und $\varrho^{\prime}$ die in Abschnitt 3.3 mit $\varepsilon=0$ eingefuihrte Funktion ist. Aus den Beweisen von Satz 6.1 und Folgerung 6.1 folgt die Behauptung

6.2 'Beim Galerkin-Verfahren mit.Spline-Funktionen soll der Einfachheit halber der Fall $\Gamma=\Gamma_{0}$ betrachtet werden.

Satz 6.3: Für $a, b \in C\left(\Gamma_{0}\right)$ und $A=a I+b S_{\Gamma_{0}} \in \mathscr{L}\left(L^{p}\left(\Gamma_{0}\right)\right)(1<p<\infty)$ ist das Cialerkin-Verjahren genau dann konvergent, wenn für alle $\lambda \in[-1,1]$ und $\tau \in \Gamma_{0}$ die Bedingung $a(\tau)+b(\tau) \lambda \neq 0$ erfüllt ist.

Beweis: Weil $y_{n}=L_{n} y \rightarrow y$ und $L_{n} A L_{n} \rightarrow A$ gilt, genügt es, die Stabilität der Folge $\left\{L_{n} A \mid s_{n}\right.$ d $\}$ zu untersuchen. Aufgrund von (4.9) muB nur die Stabilität der Folge $\left\{\left(\alpha^{-1}\right)_{n}^{\wedge}\left(a_{n}+b_{n} \hat{\kappa}_{n}\right) \hat{\alpha}_{n}\right\}$ gezeigt werden, wobei $\%:=\alpha^{-1} \beta$ gesetzt wird. J)ie Behauptung des Satzes ergibt sich nun genau wie im Beweis von Satz 6.1

Analog zum vorigen Satz und Folgerung 6.1 zeigt man

Satz 6.4: Die Funktionen $a, b \in P C\left(\Gamma_{0}\right)$ seien außerhalb der Menge $\{\exp (i \pi(2 k+1)\}$ $\left.\left.n_{0}\right): k=0, \ldots, n_{0}-1\right\}$ stetig; und es sei $d=0$. Außerdem sollen nur solche Unterteilungen in $n$ Teilintervalle und solche Spline-Räume $S_{n}{ }^{0}$ betrachtet werden, für die $n$ ein ungerades Vielfaches von $n_{0}$ ist. Dann ist das Galerkin-Verfahren für $A=a I+b S$. $\in \mathscr{L}\left(L^{p}\left(\Gamma_{0}\right)\right)(1<p<\infty)$ mit Ansatzfunktionen aus $S_{n}{ }^{0}$ genau dann konvergent, wenn für alle $t \in \Gamma_{0}$ entweder die Halbgerade $(-\infty, 0]$ den Kreisbogen $K$ aus $(0.2)$ nicht schneidet oder $(-\infty, 0)$ den Bogen $K$ in zwei inneren Punkten schneidet.

Bemerkung 6.2: Wie man bereits im Falle der Multiplikationsoperatoren sehen kann, garantiert die Bedingung des letzten Satzes nicht die Konvergenz des Galerkin-Verfahrens mit Splines aus $S_{n}{ }^{d}(d>0)$. Für diesen Fall erscheint es natïrlich, analog $z u$ [14] die verallgemcinerten Spline-Räume ' $P S_{d}{ }_{d}$ einzuführen. Es läßt sich zeigen, daß die Bedingung aus Satz 6.4 für die $L^{p}$-Konvergenz des Galerkin-Verfahrens mit Ansatzfunktionen sowohl aus $S_{n}{ }^{d}$ als auch aus $P S_{d}(d>0)$ notwendig ist. Im Falle $p=2$ ist die Bedingung des Satzes 6.4, d. h.

$$
\mu\left(d^{-1} c\right)(t+0)+(1-\mu)\left(d^{-1} c\right) \cdot(t-0) \notin(-\infty, 0] \quad \text { für aHe } \quad t \in \Gamma, \quad \mu \in[0,1],
$$

eine notwendige und hinreichende Bedingung für die $L^{2}$-Konvergenz des Cialerkin-Verfahrens mit Ansatzfunktionen aus $P S_{d}(d \geqq 0)$ (siehe [14]). 
6.3 Als letztes Verfahren soll die trigonometrische Kollokation betrachtet werden. Bei dieser Methode ist das Kollokationssystem äquivalent zu $M_{n} A x_{n}=M_{n} y$ (vgl. $(5.5))$.

Satz 6.5: Für $c, d \in \overline{P C}\left(\Gamma_{0}\right)$ und $A=c P_{r_{0}}+d Q_{\Gamma_{0}} \in \mathscr{L}\left(L^{p}\left(\Gamma_{0}\right)\right)(1<p<\infty)$ ist das trigonometrische Kollokationsverfahren genau dann stabil, wenn $A$ und $A_{-1}=c Q_{\Gamma_{0}}$ $+d P_{\Gamma_{0}}$ im Raum $L^{p}\left(\Gamma_{0}\right)$ invertierbar sind.

Beweis: Es sei $\alpha$ die charakteristische Funktion des Kreisbogens von 1 nach -1 auf $\Gamma_{0}$ und $\beta=\dot{1}-\alpha$. Dann besitzt $M_{n} P_{\Gamma_{0}}\left|T_{n}=P_{\Gamma_{0}}\right| T_{n}$ bezüglich $\left\{t^{j}\right\}_{j-r}^{r}$ die Matrix $\alpha_{n}$ und beziiglich $\left\{\psi_{k}\right\}_{k=-1}^{r}$ die Matrix $\hat{\alpha}_{n}$. Der Operator $\left.M_{n} A\right|_{T_{n}}$ hat beziiglich $\left\{\psi_{k}\right\}$ die Gestalt $c_{n} \hat{x}_{n}+d_{n} \hat{\beta}_{n}$, und aus Iemma 5.1 folgt die Behauptung (vgl. (2.2))

Die Bemerkung 6:1 gilt auch für die trigonometrische Kollokation.

Alle Resultate dieser Arbeit, die sich auf Näherungsverfahren für den Operator $A=a I$ $+6 S_{\Gamma}$ beziehen, lassen sich ohne Schwierigkeiten auf Operatoren der Form $A+T$ übertragen, wobei $T$ kompakt ist. Außerdem kömen mit der benutzten Technik auch Systeme von singulären Integralgleichungen betrachtet werden. Aus Lemma 5.1 lassen sich auch Stabilitïtskriterien für die Teilgebietsmethode (vgl. [6]) oder für Quadraturformelmethoden ableiten. Unter Ausnutzung der Approximationseigenschaft und der inversen Eigenschaft für SplineFunktionen $b \% w$. trigonometrische Polynome erhält man die Stabilität der Verfahren in bestimmten Sobolev-Räımen sowie asymptotische Konvergenzabschätzungen (vgl. [2, 181).

\section{LITERATUR}

[1] ArNOLD, D. N.: A spline-trigonometric Galerkin method and an exponentionally convergent boundary integral method. Math. Comp. 41 (1983), 383-397.

[2] ARNOLD, D. N., and W. L. WENDLAND: The convergence of spline collocation for strongly elliptic equations on curves. Numer. Math. 46 (1985); $317-341$.

[3] Aubis, J.-P.: Approximation of elliptic boundary value problems. New York-LondonSidney-Torónto: Wilcy-Interscience 1972.

[4] DE Book, C.: A bound on the $L_{\infty}$-norm of $L_{2}$-approximation by splines in terms of a global mesh ratio. Wath. Comp. 30 (1976), $765-771$.

[5] DE BOor, C.: The quasi-interpolant as a tool in elementary polynomial spline theory. In: Approximation theory. Proc. Int. Symp., Austin 1973 (Ed.: G. G. Lorestz). New YorkLondon: Academic Press 1973.

[6] Elschner, J., Prössdora, S., Rathsfeld, A., and G. Schmidt: Spline approximation of singular integral equations. Demonstratio Math. 18 (1985), 661-667.

[7] Elschner, J., and G. Schmidt: On spline interpolation in periodic Sobolev spaces. Preprint P. Mlath-01/83. Berlin: Akad. Wiss. 1984.

[8] Goch вев, I. Z., und I. A. Feldman: Filtungsgleichungen und Projektionsverfahren zu ihrer Lösung. Berlin: Akadémie-Verlag 1974.

[9] Gohвerg, I., und N. KRUPNik: Einführung in dic Theorie der ciridimensionalen singulären Integraloperatoren. Basel-Boston-Stuttgart: Birkhäuser Verlag 1979.

[10] Junghanss, P.: Kollokationsverfahren zur näherungsweisen Lösung singulärer Integralgleichungen mit unstetigen Koeffizienten. Math. Nachr. 102 (1981), 17-24.

[11] Junghavys, P., and B. Srbermann: Local theory of the collocation method for the approximate solution of singular integral equations. Int. Equ. Op. Theory 7 (1984), $791-807$.

[12] Nedмark, M. A.: Normierte Algebren. Berlin: Dt. Verlag Wiss. 1959 (2;, überarbeitete Auflage in Vorbereitung).

[13] Prössdorf, S., and A. Rathsfeld: A spline collocation method for singular integral equations with piecewise continuous coefficients. Int. Equ. Op. Theory 7 (1984), 537-560.

[14] Prössidonf, S., and A. RathSFELd: On spline Galerkin methods for singular integral equations with piecewise continuous coefficients. Numer. Math. 48 (1986), 99-118. 
[15] Prössdonf, S., and G. Schмrnt: A finite element collocation method for singular integral equations. Math. Nachr. 100 (1981), 33-60.

[16] Prössdonf, S., und. B. Silbermans: Projektionsverfahren und die näherungsweise Lösung singulärer Gleichungen (Teubner-Text zur Mathematik: Bd. 12). Leipzig: BSB B. G. Teubner Verlagsgesellischaft 1977.

[17] RathSFELD, A.: Reduktionsverfahren für singuläre Integralgleichungen mit stückweise stetigen Koeffizienten. Math. Nachr. 127 (1986), 125-143.;

[18] SснміDт, G.: On $\varepsilon$-collocation for pseudodifferential equations on a closed curve. Nath. Nachr. 126 (1986), 183-196.

[19] Schmidt, G.: On spline collocation methods for boundary integral equations in the plane. Math. Meth. in Appl. Sci. 7 (1985), 74-89.

[20] Schmidr, G.: The Convergence of Galerkin and Collocation Methods with Splines for Pseudodifferential Equations on Closed Curves. Z. Anal. Anw. 3 (1984), $371-384$.

[21] Trieber, H.: Interpolation theory, function spaces, différential operators. Berlin: Dt. Verlag Wiss./Amsterdam-New York-Oxford: North-Hollaud Publ. Comp. 1978.

[22] WhNDLAND, W. L.: On the spline approximation of singular integral equations and onedimensional pseudo-differential equations on closed curves. In: Numerical solution of singular integral equations. Proc. IMACS Int. Symp. Lehigh Univ. Bethlehem, Pennsylvania (USA), June.21-22, 1984 (Eds: A: Gerasovis and Ŕ. VichnevetSkY).

[23] Zygmund, A: : Trigonometric Series, Vol. 11. Cambridge: University Press 1959.

Manuskripteingang: 01. 07. 1985, in revidierter Fassung 24.04.1986

\section{VERFASSER:}

Prof. Dr. S. Prössdorf und A. Ratrisfald

Karl:Weierstraß,-Institut für Mathematik

der Akademie der Wissenschaften

Mohrenstr. 39

DDR - 1086 Berlin 